\title{
A long hard look at the minimum state of PG 2112+059 with XMM-Newton
}

\author{
N. Schartel ${ }^{1}$, P. M. Rodríguez-Pascual ${ }^{1}$, M. Santos-Lleó ${ }^{1}$, E. Jiménez-Bailón ${ }^{2}$, L. Ballo ${ }^{1,3}$, and E. Piconcellii \\ 1 XMM-Newton Science Operations Centre, ESA, Villafranca del Castillo, Apartado 78, 28691 Villanueva de la Cañada, Spain \\ e-mail: Norbert.Schartel@sciops.esa.int \\ 2 Instituto de Astronomía, Universidad Nacional Autónoma de México, Apartado Postal 70-264, 04510-Mexico DF, México \\ 3 Instituto de Física de Cantabria (CSIC-UC), 39005 Santander, Spain \\ 4 Osservatorio Astronomico di Roma (INAF), via Frascati 33, 00040 Monteporzio Catone, Italy
}

Received 25 April 2009 / Accepted 28 November 2009

\section{ABSTRACT}

\begin{abstract}
Aims. Our observational aim is to perform a long X-ray observation of the quasar PG $2112+059$ in its low or minimum state. Starting form this very peculiar emission state, we intend to constrain the intrinsic emission mechanism by comparing new and old data, corresponding to different source states.

Methods. XMM-Newton successfully detected the minimum state of PG $2112+059$ during a short snapshot observation and performed a long follow-up observation. The high signal-to-noise spectra are modelled assuming different emission scenarios and compared with archival spectra taken by XMM-Newton and Chandra.

Results. The PG 2112+059 X-ray spectra acquired in May 2007 allowed the detection of a weak iron fluorescent line, which is interpreted as being caused by reflection from neutral material at some distance from the primary X-ray emitting source. The X-ray spectra of PG 2112+059 taken at five different epochs during different flux states can be interpreted within two different scenarios. The first consists of two layers of ionised material with column densities of $N_{\mathrm{H}} \sim 5 \times 10^{22} \mathrm{~cm}^{-2}$ and $N_{\mathrm{H}} \sim 3.5 \times 10^{23} \mathrm{~cm}^{-2}$, respectively. The first layer is moderately ionised and its ionisation levels follow the flux changes, while the other layer is highly ionised and does not show any correlation with the flux of the source. The spectra can also be interpreted assuming reflection by an ionised accretion disk seen behind a warm absorber. The warm absorber ionisation is consistent with being correlated with the flux of the source, which provides an additional degree of self-consistency with the overall reflection-based model. We explain the spectral variability with light bending according to the models of Miniutti and Fabian and constrain the black hole spin to be $a / M>0.86$. Both scenarios also assume that a distant cold reflector is responsible for the $\mathrm{Fe} \mathrm{K} \alpha$ emission line.

Conclusions. Light bending provides an attractive explanation of the different states of PG $2112+059$ and may also describe the physical cause of the observed properties of other X-ray weak quasars. The observations of PG $2112+059$ in different states provide valuable constraints, although are unable to break the degeneracy between complex absorption scenarios and reflection from an ionised disk.
\end{abstract}

Key words. galaxies: active - quasars: individual: PG 2112+059

\section{Introduction}

$\mathrm{X}$-ray weak or soft X-ray weak quasars are characterised by an X-ray emission lower, by a factor of 10-30, than expected based on their optical-UV emission (Laor et al. 1997; Wang et al. 1996; Elvis \& Fabbiano 1984). About 10\% of bright, optically selected quasars belong to this subclass (Laor et al. 1997; Brandt et al. 2000; Gibson et al. 2008).

Analysing the Boroson \& Green (1992) sample of quasars, Brandt et al. (2000) found a correlation between soft X-ray weakness and C IV absorption equivalent width $(E W)$. This correlation implies that absorption is the primary cause of soft $\mathrm{X}$-ray weakness, which is in general agreement with models connecting orientation and absorption strength. However, a uniform screen of material that completely covers both the X-ray and ultraviolet emission sources is in disagreement with the shape of the correlation (Brandt et al. 2000). Since the ultraviolet absorption is produced by a resonance line while the $\mathrm{X}$-ray absorption by bound-free edges, the optical-to-X-ray spectral index, $\alpha_{\mathrm{OX}}$ is expected to be practically independent of the C IV $E W$ up to a column density corresponding to $\tau_{\mathrm{bf}} \sim 1$. Above this value, a very rapid decline in $\alpha_{\mathrm{OX}}$ and a slow increase in C IV $E W$ is expected. We calculate the indices following Strateva et al. (2005), taking the fluxes at $2500 \AA$ and $2 \mathrm{keV}$ in the rest frame of the quasar, $\alpha_{\mathrm{OX}}=\log \left(F_{2 \mathrm{keV}} / F_{2500} \AA\right) / \log \left(v_{2 \mathrm{keV}} / v_{2500} \AA\right)$. The correlation discovered between $\alpha_{\mathrm{OX}}$ and C IV $E W$ instead exhibits a gradual increase in the C IV EW associated with a gradual decrease in $\alpha_{\text {OX }}$ (compare Fig. 4 in Brandt et al. 2000). This implies that either the UV and X-ray absorbers are distinct or the $\mathrm{X}$-ray weakness is produced mainly by a peculiar property of the nuclear emission such as extreme variability. In addition, Brandt et al. (2000) noted several differences between X-ray weak and "normal" quasars such as low [O III] luminosities and equivalent widths, distinctive $\mathrm{H} \beta$ profiles and a location toward the weak [O III] end of the Boroson \& Green eigenvector 1, which may be indicative of a high mass accretion rate relative to the Eddington limit.

Brandt et al. (2000) speculated that with increasing inclination angle we may observe quasars, $\mathrm{X}$-ray weak quasars, broad absorption line (BAL) quasars and type 2 quasars, which was also proposed by Elvis (2000). This idea has been supported 
by various observations. On the one hand, X-ray weak quasars were recognised as being BAL or mini-BAL quasars (Gallagher et al. 2001; Sulentic et al. 2006). On the other hand, X-ray observations indicated high column densities for many X-ray weak quasars (Gallagher et al. 2001, 2004; Brinkmann et al. 2004; Piconcelli et al. 2004; Schartel et al. 2005).

Various results challenge this picture and indicate greater complexity. In addition X-ray weakness is much less characteristic of BAL quasars class than previously understood (Giustini et al. 2008; Blustin et al. 2008; Fan et al. 2009; Gibson et al. 2009). Some $X$-ray weak quasars exhibit significant variability being sometimes X-ray weak and sometimes not (Ballo et al. 2008). The variability is often correlated with a significant change in the spectral properties of the continuum emission.

Grupe et al. (2007, 2008b) discussed whether varying absorbing column densities could explain the variable X-ray weakness and spectral properties found for WPVS 007. During the ROSAT All Sky Survey, this source was observed in an X-ray high state, but all subsequent observations (between 1993 and 2007) detected fainter X-ray emission. WPVS 007 is particularly interesting because its FUSE spectrum obtained in 2003 exhibits a BAL outflow that is not present in the HST FOS spectrum acquired in 1996 (Leighly et al. 2009). An X-ray weak continuum or even an intrinsically X-ray weak spectrum in combination with the X-ray absorption implied by the UV data provides the most probable interpretation of the observed spectrum (Leighly et al. 2009). Another X-ray weak quasar, PHL 1811, seems to be an intrinsically weak narrow-line quasar (Leighly et al. 2007a). Althought its X-ray spectral slopes are consistent with the values commonly observed in narrow-line Seyfert $1 \mathrm{~s}$, its X-ray spectra show no evidence of absorption and its UV spectra lack absorption lines (Leighly et al. 2007b). The narrow line quasar PHL 1092 exhibits a dramatic variability making it one of the most extreme X-ray weak quasars (Miniutti et al. 2009). The inferred steep power-law index and its persistent UV flux imply that an absorption event is unlikely, suggesting instead that a transient weakening or disruption of the X-ray emitting corona is responsible.

Schartel et al. (2007) and Ballo et al. (2008) discussed reflection-dominated scenarios for low states of PG 2112+059 and PG 1535+547, respectively. Similar scenarios were proposed by Gallo (2006) for narrow-line Seyfert 1s, by Grupe et al. (2008a) for the historical low state of Mrk 335, and by Vignali et al. (2008) for the narrow-line quasar PG 1543+489. The most impressive case of a reflection-dominated AGN is the narrowline Seyfert 1 1H 0707-495. Its X-ray spectra show a sharp spectral drop, which Fabian et al. $(2002,2004)$ interpreted in terms of relativistically blurred ionised reflection from the accretion disk. A four-orbit-long XMM-Newton observation of 1H0707-495 in 2008 detected broad lines from ionised iron $\mathrm{K}$ and iron $\mathrm{L}$. The latter, observed for the first time in this type of observation, appears 20 times weaker than the iron $\mathrm{K}$, in agreement with predictions based on atomic physics. In addition, Fabian et al. (2009) measured for the first time the expected frequency-dependent time-lags between the power-law and the reflection component. In spite of these developments, it is plausible that in some cases low states and X-ray weaknesses are caused by suppressed continuum emission in the direction of our line of sight, as proposed by the light-bending model of Miniutti \& Fabian (2004). In this model, the primary X-ray emitting source is located above the supermassive black hole and moves up and down. If the source is within a few Schwarzschild radii of the black hole, then a substantial part of the primary continuum emission may bend onto the accretion disk. Therefore, observers see suppressed continuum emission and an enhanced reflection component at the same time, which increases the probability of detecting emission from the accretion disk.

To study this possibility in more detail, we proposed a $200 \mathrm{ks}$ deep X-ray observation of PG $2112+059$ in a low or deep minimum state (Schartel et al. 2007). As the source is highly variable, we proposed to complete a short snapshot observation followed by a targeted deep observation only if the source was detected at a flux level comparable to that measured in November 2005.

In Sect. 2, we characterise PG 2112+059 and summarise its observation history in X-rays. The XMM-Newton observations and the subsequent data reduction are presented in Sect. 3. We shifted the observation and extraction tables to the Appendix for the convenience of our reader. The analysis of the timing behaviour of both the optical and X-ray data follows in Sect. 4. In Sect. 5, we provide the X-ray spectral analysis followed by the discussion in Sect. 6 and our general conclusions in Sect. 7.

\section{PG 2112+059}

The quasar PG 2112+059 was recognised to be such within the Palomar bright quasar survey (Schmidt \& Green 1983). The source has a redshift of $z=0.456$ (Véron-Cetty \& Véron 2000). Based on the Hubble Space Telescope spectrum (Jannuzi et al. 1998), Gallagher et al. (2001) could classify PG 2112+059 as a BAL quasar. The supermassive black hole of PG 2112+059 has one of the highest masses of all Palomar quasars with $\log M_{\mathrm{BH}} / M_{\text {solar }}=9.0 \pm 0.1$ (Vestergaard \& Peterson 2006).

PG 2112+059 was observed by ROSAT in 1991, by ASCA in October 1999 (Gallagher et al. 2001), by Chandra in September 2002 (Gallagher et al. 2004), and twice by XMM-Newton in May 2003 and November 2005 (Schartel et al. 2005, 2007). Based on the ROSAT data, Wang et al. (1996) first measured a low optical-to-X-ray spectral index as is characteristic of X-ray weak quasars. The observations found dramatic variability in the X-ray energy range. The highest flux was that detected by ASCA, which was approximately a factor of four higher than in the earlier ROSAT observation as well as in the later Chandra observation. In May 2003, the source was in an intermediate flux state and in November 2005 a deep minimum state.

The ASCA spectrum of PG $2112+059$ detected clear evidence of absorption but the photon statistics were insufficient for any accurate characterisation. The Chandra spectrum is incompatible with neutral absorption completely covering the source. The spectrum can be described statistically assuming an ionised absorber as well as a partially covering neutral absorber (Gallagher et al. 2004), where both absorption scenarios require an increase in the absorbing column density between 1999 and 2002. Schartel et al. (2007) interpreted the two XMM-Newton observations in the context of light blending (Miniutti \& Fabian 2004) but could not exclude complex absorption scenarios involving two layers of absorbing material with different ionisation states.

\section{Observations and data reduction}

In 2007, XMM-Newton (Jansen et al. 2001) observed PG 2112+059 four times. First, to determine the current state of the quasar, a short ( $25 \mathrm{ks})$ snapshot-type observation (observation identifier (ObsId) 5005006) was performed on the 3 May 2007. Since this observation detected the quasar in the targeted low state, XMM-Newton pointed towards PG 2112+059 for two adjoining revolutions with the maximum possible exposure time of $\sim 100 \mathrm{ks}$ each from the 19 May 2007 onwards 
Table 1. Optical variability of PG 2112+059: OM fluxes.

\begin{tabular}{lccccccc}
\hline \hline \multirow{2}{*}{ Filter } & Effective & \multicolumn{2}{c}{ Epoch } & \multicolumn{2}{c}{ Average flux } & Ratio \\
& Wavelength & $2003-05$ & $2005-11$ & $2007-05$ & $2007-11$ & & 4 \\
\hline$V$ & {$[\mathrm{~nm}]$} & 1,2 & 1 & 1 & 1 & 1,3 & $4.07 \pm 0.02$ \\
& $500-590$ & $22 \pm 2$ & $20.7 \pm 0.1$ & $20.0 \pm 0.1$ & $19.4 \pm 0.2$ & $20.1 \pm 0.1$ & $1.05 \pm 0.01$ \\
$U$ & $380-490$ & $31 \pm 2$ & $29.3 \pm 0.1$ & $28.0 \pm 0.1$ & $27.9 \pm 0.2$ & $28.3 \pm 0.1$ & $1.05 \pm 0.1$ \\
$U V W 1$ & $300-390$ & $37 \pm 3$ & $38.9 \pm 0.1$ & $36.4 \pm 0.1$ & $35.4 \pm 0.2$ & $36.9 \pm 0.1$ & $1.10 \pm 0.01$ \\
$U V M 2$ & $240-360$ & & $46.7 \pm 0.2$ & $43.4 \pm 0.1$ & $42.4 \pm 0.2$ & $43.7 \pm 0.1$ & $1.10 \pm 0.01$ \\
$U V W 2$ & $180-270$ & & $60.7 \pm 0.8$ & $55.0 \pm 0.4$ & $54.3 \pm 0.7$ & $55.7 \pm 0.3$ & $1.12 \pm 0.03$ \\
& & $63.8 \pm 1.3$ & $56.6 \pm 0.8$ & $54.9 \pm 1.2$ & $57.5 \pm 0.6$ & $1.16 \pm 0.05$ \\
\hline
\end{tabular}

Notes. (1) Flux in units of $10^{-16} \mathrm{erg} / \mathrm{cm}^{2} / \mathrm{s} / \AA .{ }^{(2)}$ Estimated based on optical grism observation. ${ }^{(3)}$ Average flux excluding $2003-05$ data. (4) Maximum to minimum flux ratio excluding 2003-05 data.

(ObsId 5005007 and 5005008). About half a year later, during the autumn visibility window of the target, a fourth observation (ObsId: 5005009) was performed to test whether the quasar remained in the low state. This observation started on the 5 November and accumulated photons for $53 \mathrm{ks}$.

To take full advantage of the calibration progress and allow an unbiased comparison of all XMM-Newton observations of PG 2112+059, we re-extracted the data taken in May 2003 (ObsId: 150610201) and the data taken in November 2005 (ObsId: 3003102). Details of these observations are given in Schartel et al. (2005, 2007). In addition we considered the spectrum taken by Chandra in September 2002 (ObsId 3011). Gallagher et al. (2004) provide details of this observation.

XMM-Newton carries three scientific instruments that observe simultaneously: the Reflection Grating Spectrometer (RGS; Brinkman et al. 2001), the European Photon Imaging Camera (EPIC), and the Optical Monitor (OM; Mason et al. 2001). EPIC consists of three CCD cameras: the pn-camera (Strüder et al. 2001) and two MOS-cameras (Turner et al. 2001). All EPIC exposures of PG $2112+059$ were taken in full frame mode with different optical blocking filters in the light path. OM observed in an "science user defined" image mode with different filters or grisms in the optical light path. The details of the exposures are provided in Appendix A.

We processed the data with the XMM-Newton Science Analysis System (SAS) v. 7.1 (linux; compare Loiseau et al. 2006) with the calibration files generated between August and November 2007. Neither the pipeline products provided by the Survey Science Centre (Watson et al. 2001) nor our visual inspection of the processed RGS events in the dispersion-crossdispersion-plane allowed a detection of the source with RGS.

We processed the EPIC and OM data exactly as described in Schartel et al. (2007), applying the screening procedure for time ranges with low radiation background level according to Piconcelli et al. (2005). The details of the extraction and its results are described in Appendix B.

The Chandra data reduction was performed using the Ciao 3.4 version applying the calibration according to CALDB 3.4.0 (May 2007). Source and background spectra, and response matrices were produced using the psextract ciao task. The source spectrum was extracted from a circular region centred on the maximum emission with a $4.92^{\prime \prime}$ (10 pixels) radius. The background spectrum was extracted using five different regions free of any contaminating source. They are all located in the same CCD with a distance of $25-40^{\prime \prime}$ to the source. The Chandra spectrum was binned to ensure at least 20 counts per energy bin.

\section{Timing analysis}

\section{1. $O M$}

To test for optical variability on short time scales, we face the difficulty that within each observation only a few exposures for each of the optical filters were performed. Therefore, for each filter we divided the maximal flux by the minimal one and propagated the errors accordingly. All results are in agreement with 1 within the errors. Consequently, we exclude optical variability being present in the individual observations.

Table 1 gives the mean fluxes as measured with the different OM filters for May 2003, Nov. 2005, and May and Nov. 2007. It also provides per filter the ratio of the maximum to minimum flux observed between 2005 and 2007 (i.e., excluding 2003 data), as well as the average flux. The optical fluxes measured in 2007 are significantly lower than in 2005, the amplitude of the variation being larger at shorter wavelengths. The fluxes also appear to decrease between May and November 2007, although the statistical evidence is much weaker. The ratios of maximum to minimum fluxes demonstrate that the variation correlates with wavelength.

During the first XMM-Newton observation, performed in May 2003, only OM grism exposures were performed. To estimate the broad-band flux, we folded the flux observed in the optical grism through the effective area of the filters. The results are provided in Table 1. The quoted fluxes are uncertain and the errors are larger than the quoted statistical extraction errors. The presence of spectra from other sources complicates the extraction of the quasar grism spectrum and the result still depends on the selected source and background extraction regions. So, the maximal conclusion from the optical grism in 2003 is that the flux in that epoch did not differ to within 10-20\% from the flux measured in later observations.

\subsection{X-ray}

To test for X-ray variability within the individual observations, we created light curves for different time bins and energy ranges for each of the four PG $2112+059$ observations performed in 2007. The light curves were created from the unscreened events collected by the pn-camera as this camera has the largest effective area of all X-ray observing instruments of XMM-Newton. We extracted the source and background photons from the same sky area as used for the spectral analysis (specified in the Appendix, Table B.1, for each observation). We produced light curves with time bins of $5000 \mathrm{~s}, 10000 \mathrm{~s}, 15000 \mathrm{~s}$ and $20000 \mathrm{~s}$ for the 0.35 to $0.5 \mathrm{keV}, 0.5$ to $2.0 \mathrm{keV}, 2$ to $10 \mathrm{keV}$, 


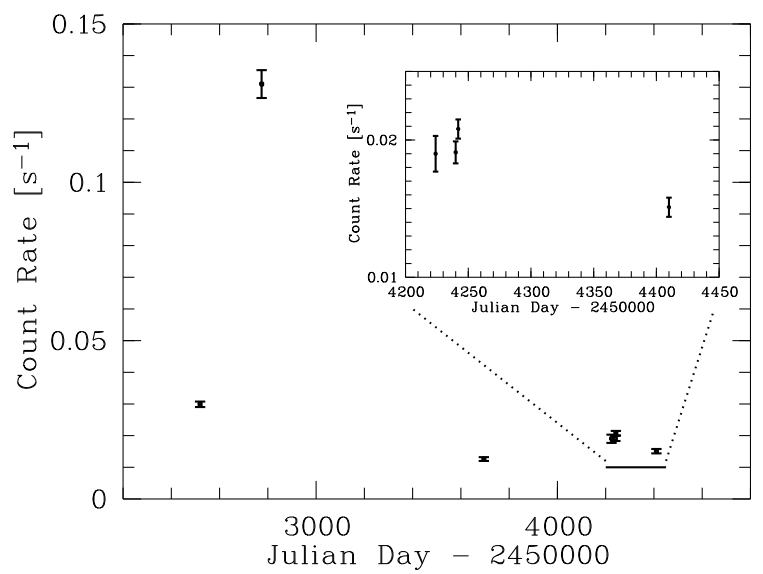

Fig. 1. X-ray variability of PG 2112+059 between September 2002 and November 2007 is shown. The image shows the count rate measured by the pn camera as a function of the Julian date. The count rate measured with Chandra in 2002 was converted to a corresponding pn count rate (first data point). The inlay shows the count rates collected in 2007.

and 0.3 to $12 \mathrm{keV}$ energy bands. The softest band in combination with $5000 \mathrm{~s}$ or $10000 \mathrm{~s}$ bin size shows too few counts per bin to permit further analysis. Inspecting the light curves, we found no obvious variability pattern and we estimate the variability to be within a range of $20 \%$.

PG $2112+059$ shows a pronounced variability over longer time scales. The pn count rates of the observations discussed in this paper are plotted in Fig. 1, where the count rate observed by Chandra was converted based on a spectral fit. The count rates corresponding to the observation taken in 2007 are shown in detail in the inlay. After the 2003 high state, PG 2112+059 has always been observed during low states showing a flux a factor from 7 to 10 lower than in a high state. The flux before the high state is about $30 \%$ higher than the flux observed after it. However, it should be noted that high states in-between the measurements cannot be excluded.

\section{3. $\alpha_{\mathrm{OX}}$}

As mentioned, we calculated $\alpha_{\mathrm{OX}}$ following Strateva et al. (2005) by taking the fluxes at $2500 \AA$ and $2 \mathrm{keV}$ in the rest frame of the quasar. The X-ray flux was determined using fit 4 provided in Table 5. Both the X-ray and optical data were corrected for Galactic absorption. For the optical data, we applied the extinction curve provided by Cardelli et al. (1989). We did not correct the optical data for the contribution of the host galaxy, thus the nuclear UV flux could be lower than the value estimated here.

The relation between rest-frame UV and soft X-ray AGN emission, and its dependence on redshift and/or optical luminosity was investigated several times. Most studies detected no evidence of a redshift dependence, while the X-ray emission was found to be correlated with the UV emission such that $\alpha_{\text {OX }}$ decreases as the UV emission increases (e.g., Vignali et al. 2003; Strateva et al. 2005; Steffen et al. 2006; Just et al. 2007). Strateva et al. (2005) combined radio quiet sources from the SDSS, a heterogeneous low-redshift Seyfert 1 sample, and a heterogeneous high-redshift sample. They found a correlation between the monochromatic luminosities at $2500 \AA$ and $2 \mathrm{keV}$. The broadband spectral index is anticorrelated with the restframe monochromatic UV luminosity. PG 2112+059 appears to be under-luminous in the X-ray band compared to optically selected sources in the same bin of UV luminosity. In Fig. 2, we

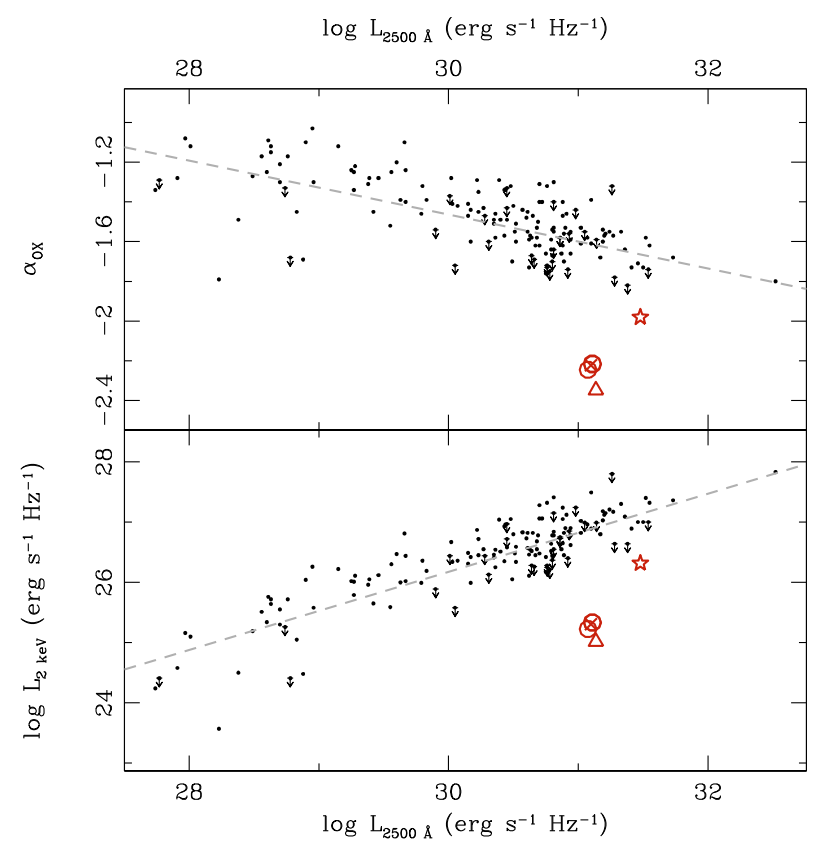

Fig. 2. The dependence on the $2500 \AA$ monochromatic luminosity of $\alpha_{\mathrm{OX}}$ (upper panel) and $L_{2 \mathrm{keV}}$ (lower panel) is shown in comparison with the values obtained for PG 2112+059. Black-filled circles mark data from Strateva et al. (2005) for their "main" SDSS sample (155 objects with $0.1 \lesssim z \lesssim 4.5$ ); arrows indicate upper limits to the X-ray detection. Dashed lines are the best-fit linear relations for their combined sample ("main" SDSS sample plus high-redshift sample plus Seyfert 1 sample). The values derived for PG 2112+059 are plotted in grey (red in the colour edition). The star labels the observation from May 2003, the triangle from November 2005, the circles from May 2007, and the " $x$ " from November 2007.

compare the UV and X-ray luminosities, and $\alpha_{\mathrm{OX}}$ with the trend found by Strateva et al. (2005) for their sample of 155 optically selected active galactic nuclei.

We also determined the absorption-corrected optical-to$\mathrm{X}$-ray spectral index. We based our calculation on the most accurately estimated parameters of fit 3 and 4 (Table 5). The resulting values of $\alpha_{\mathrm{OX}}<-1.7$ imply that PG $2112+059$ was observed while intrinsically X-ray weak in each of the XMM-Newton observations.

\section{X-ray spectral analysis}

We performed the spectral analysis as described by Schartel et al. (2007). Following the standard calibration recommendations (Guainazzi 2009), we analysed the MOS spectra over the energy range from $0.2 \mathrm{keV}$ to $10.0 \mathrm{keV}$ and the pn spectra over the 0.2 to $12.0 \mathrm{keV}$ energy range.

The count rates of all three observations from May 2007 agree with those of a constant source, and a visual inspection showed no indication of differences in the spectral slope. We therefore added the spectra obtained for each instrument and calculated the corresponding effective areas and detector response matrices giving weight according to the accumulated exposure time. In addition, we added the spectra obtained for MOS1 and MOS2 for all observations and calculated the corresponding auxiliary files by applying the same weighting with exposure time. As reported in Schartel et al. (2007) for the 2005 observation, in all cases the accumulated counts at low energies were 
too few to take advantage of the differences in effective area associated with the different optical blocking filters.

Reflecting the high number of collected counts, we binned the May 2007 combined pn spectrum to achieve a signal-tonoise ratio of at least 6 . All other pn and combined MOS spectra were binned to reach a signal-to-noise ratio of at least 5 . In all cases, we limit the binning to three bins per spectral resolution element. We used XSPEC 12.5.0ac (Linux version, Arnaud 2009) in the spectral analysis that estimates spectral parameters with the modified minimum $\chi^{2}$ method (Kendall \& Stuart 1973). Errors in estimated parameters are provided for the $90 \%$ confidence region for a single interesting parameter $\left(\Delta \chi^{2}=2.7\right.$, Avni 1976). All modelling was performed based the assumption of Galactic foreground absorption with an equivalent column density of $N_{\mathrm{H}}=6.22 \times 10^{20} \mathrm{~cm}^{-2}$ (Kalberla et al. 2005). For the plasma emission and photoelectric absorption, we assumed solar abundances as determined by Anders and Ebihara (1982) and a photoelectric cross-section according to Verner et al. (1996). We assumed a cosmology with $\Omega_{\mathrm{M}}=0.27, \Omega_{\Lambda}=0.73$ and $H_{0}=70 \mathrm{~km} \mathrm{~s}^{-1} \mathrm{Mpc}^{-1}$ (Spergel 2008).

The signal-to-noise ratios obtained for the November 2007 spectra are too low to allow us to set constraints of the physical emission mechanisms. We therefore focus in the following on the added spectra of the May 2007 observation and use the November spectra only to check for consistency and in the joint modelling of all the obtained spectra.

\subsection{Spectra taken in May 2007}

For the spectral analysis, we fitted the pn spectrum and the MOS spectrum, obtained by adding the MOS1 and MOS2 spectra, together constraining all the free parameters to have the same value for the two data sets. Reflecting differences in the absolute calibration of the two instruments (Guainazzi 2009), the model for the MOS spectrum was multiplied by 1.07 .

As for the spectra measured during observations in November 2005 (Schartel et al. 2007), the spectra of PG 2112+059 taken in May 2007 is rather hard without showing a pronounced narrow iron $\mathrm{K} \alpha$ fluorescent emission line as expected for heavily absorbed sources. For illustration, we show in Fig. 3 the pn and MOS count spectra, and for comparison the counts expected for a canonical power-law model $(\Gamma=1.89$, Piconcelli et al. 2005) absorbed by Galactic absorption with arbitrary normalisation. A canonical power-law model may provide a first-order good approximation for energies $>5 \mathrm{keV}$, implying an enormous deficit at lower energies. In addition a canonical power-law may provide a first-order good approximation of the soft energy range $(0.2$ to $2 \mathrm{keV})$ that fails in this case to account for the hard band: in particular, the data show a large excess compared with this model in the 2 to $5 \mathrm{keV}$ energy range. Starting from these considerations and taking into account the results obtained from previous observations, we attempt to explain the spectra within an absorption scenario and compare the results with a model based on reflection within an ionised disk.

\subsubsection{Simple absorption models}

We cannot describe the spectra with a power law source observed behind a layer of neutral $\left(\chi^{2}=378.4\right.$, d.o.f. $\left.=102\right)$ or ionised $\left(\chi^{2}=158.5\right.$, d.o.f. $\left.=101\right)$ material located at the redshift of the source. Within the analysis, two descriptions of the ionised absorber were used as provided in XSPEC by the models

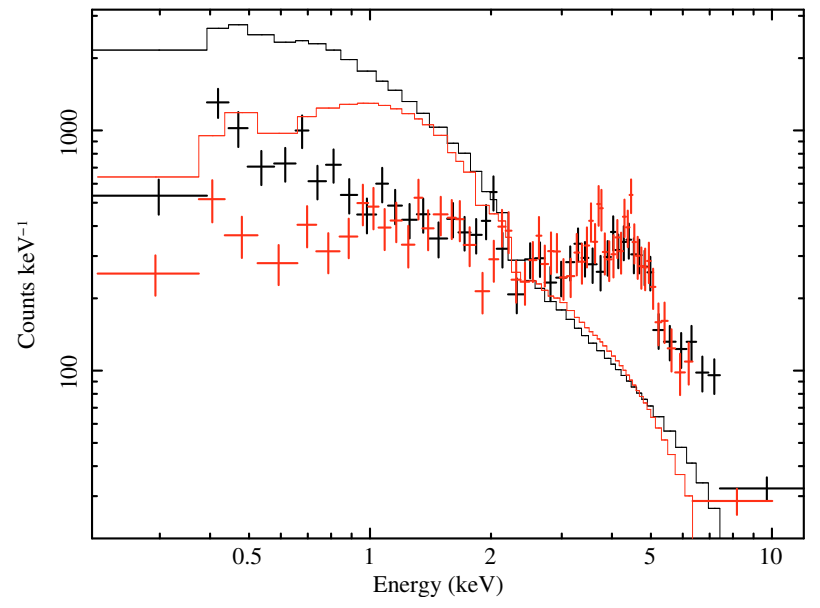

Fig. 3. The figure shows the observed (count) spectra of pn (black) and combined MOS (grey - red in the colour edition) of PG 2112+059 taken in May 2007. In addition, we show the counts expected for a canonical power-law model $(\Gamma=1.89)$ absorbed by Galactic absorption with arbitrary normalisation. In comparison to the expectation for a canonical power law, the observed spectra is very hard, which may be indicative of either a huge deficit below $5 \mathrm{keV}$ or a large excess emission in the 2 to $5 \mathrm{keV}$ energy range.

Table 2. Spectral models.

\begin{tabular}{|c|c|c|}
\hline $\begin{array}{l}\text { Model } \\
\text { label }\end{array}$ & Description and references & $\begin{array}{l}\text { XSPEC } \\
\text { name }\end{array}$ \\
\hline i & $\begin{array}{l}\text { partial covering with ionised absorber at the } \\
\text { redshift of the source (Reeves et al. 2008) }\end{array}$ & zxipcf \\
\hline$d$ & $\begin{array}{l}\text { reflection by distant neutral material } \\
\text { including fluorescence emission lines } \\
\text { (Nandra et al. 2007) }\end{array}$ & pexmon \\
\hline$p$ & power-law & pow \\
\hline$k$ & $\begin{array}{l}\text { relativistic blurring from an accretion disk } \\
\text { around a rotating Kerr black hole } \\
\text { (Dov̌ciak et al.2004) }\end{array}$ & kyconv \\
\hline$n$ & neutral absorber at the redshift of the source & zphabs \\
\hline$r$ & $\begin{array}{l}\text { reflection by a constant density illuminated } \\
\text { atmosphere (Ross \& Fabian 2005) }\end{array}$ & reflion \\
\hline
\end{tabular}

absori $^{1}$ and zxipcf $f^{2}$. To keep the discussion simple, here and in the following we refer to results obtained with zxipcf (see Table 2) only. A partially covering neutral absorber allows a marginal statistically acceptable description of the spectra $\left(\chi^{2}=\right.$ 129.2 , d.o.f. $=101)$, but shows systematic residua in the 3 to $6 \mathrm{keV}$ energy range.

\subsubsection{Complex absorption models and distant reflection}

We model the spectra assuming various combinations of different absorbers. We considered neutral and ionised material, both totally or partially covering the source. From the statistical viewpoint, all models provide an acceptable description of the spectra, but exhibit a feature at $4.4 \mathrm{keV}$ in the observers frame. The corresponding energy in the rest-frame of the quasar, $6.4 \mathrm{keV}$, equals exactly the energy of the $\mathrm{K}_{\alpha}$ fluorescence line of

\footnotetext{
${ }^{1}$ Ionised absorber model (Zdziarski et al. 1995); it is just a first approximation, the temperature being considered as an input parameter, and not calculated self-consistently by proper thermal balance.

${ }^{2}$ Model for partial covering of a partially ionised absorbing material, based on a pre-calculated grid of XSTAR photoionised absorption model (Reeves et al. 2008).
} 
Table 3. Spectral fits of the May 2007 EPIC spectra of PG 2112+059: Continuum properties.

\begin{tabular}{|c|c|c|c|c|c|c|c|c|c|}
\hline \multirow[t]{2}{*}{ Fit } & \multirow[t]{2}{*}{ Model $^{a}$} & \multicolumn{3}{|c|}{$\begin{array}{c}\text { Absorber } \\
\text { neutral }\end{array}$} & \multicolumn{2}{|c|}{$\begin{array}{l}\text { Continuum } \\
\text { power-law }\end{array}$} & \multirow{2}{*}{$\begin{array}{c}\text { Distant } \\
\text { Reflection } \\
\text { Norm } \\
{\left[\mathrm{keV}^{-1} \mathrm{~cm}^{-2} \mathrm{~s}^{-1}\right]}\end{array}$} & \multicolumn{2}{|c|}{ Statistic } \\
\hline & & $\begin{array}{c}N_{\mathrm{H}}{ }^{b} \\
{\left[10^{22} \mathrm{~cm}^{-2}\right]}\end{array}$ & $\mathrm{cf}^{c}$ & $\begin{array}{l}\xi^{d} \\
{[\log ]}\end{array}$ & $\Gamma^{e}$ & $\begin{array}{c}\text { Norm }{ }^{g} \\
{\left[\mathrm{keV}^{-1} \mathrm{~cm}^{-2} \mathrm{~s}^{-1}\right]}\end{array}$ & & $x^{2}$ & $d^{h}$ \\
\hline 1 & $i *(p+d)$ & $18.3_{-1.9}^{+2.7}$ & $0.94_{-0.02}^{+0.01}$ & $1.10_{-0.52}^{+0.45}$ & $2.08_{-0.13}^{+0.16}$ & $8.74_{-2.51}^{+2.29} 10^{-5}$ & $9.24_{-514}^{+8.03} 10^{-5}$ & 93.2 & 99 \\
\hline 2 & $i *(p+k(r)+d)$ & $0.12_{-0.02}^{+0.39}$ & $1.00_{-0.14}^{+0.00}$ & $-1.36_{-0.31}^{+3.04}$ & $1.53_{-0.10}^{+0.16}$ & $3.58_{-0.89}^{+0.74} 10^{-6}$ & $1.45_{-0.95}^{+1.17} 10^{-5}$ & 96.7 & 93 \\
\hline
\end{tabular}

Notes. Explanation of labels: ${ }^{(a)}$ the spectral models described in Table 2; ${ }^{(b)}$ equivalent column density; ${ }^{(c)}$ covering fraction; ${ }^{(d)}$ ionisation level of absorbing material $\xi=L / n r^{2}$, as defined in XSPEC; ${ }^{(e)}$ photon index of power-law continuum; ${ }^{\left({ }^{g}\right)}$ normalisation of photon flux at $1 \mathrm{keV} ;{ }^{(h)}$ degrees of freedom. The best-fit parameters for the ionised reflector, the disk, and the black hole are provided in Table 4 . All errors are provided at the $90 \%$ confidence level.

Table 4. Spectral fits of the May 2007 EPIC spectra of PG 2112+059: Ionised reflector, disk, and black hole properties.

\begin{tabular}{|c|c|c|c|c|c|c|c|}
\hline \multirow{2}{*}{ Fit } & \multirow{2}{*}{$M^{a}$} & \multicolumn{3}{|c|}{ Ionised reflector } & \multicolumn{2}{|c|}{ Disk } & \multirow{2}{*}{$\begin{array}{c}\text { Black Hole } \\
a / M^{e}\end{array}$} \\
\hline & & $\begin{array}{c}\text { Norm } \\
{\left[\mathrm{cm}^{-2} \mathrm{~s}^{-1}\right]}\end{array}$ & $\begin{array}{c}\mathrm{Fe}^{b} \\
{\left[\mathrm{erg} \mathrm{cm} \mathrm{s}^{-1}\right]}\end{array}$ & $\Xi^{c}$ & $\begin{array}{c}\text { Index }^{d} \\
\text { [degree] }\end{array}$ & Inclination & \\
\hline 2 & $k(r)$ & $1.69_{-1.25}^{+11.7} \times 10^{-6}$ & $1.74_{-0.30}^{+0.32}$ & $30.0_{-0.0}^{+17.8}$ & $3.29_{-0.58}^{+2.00}$ & $52.1_{-3.0}^{+3.1}$ & $0.93_{-0.10}^{+0.07}$ \\
\hline
\end{tabular}

Notes. The corresponding continuum properties are provided in Table 3. Explanation of labels: ${ }^{(a)}$ the spectral models described in Table 2; ${ }^{(b)}$ iron abundance relative to solar iron abundance; ${ }^{(c)}$ ionisation parameter as defined in reflion; ${ }^{(d)}$ power-law dependence of emissivity; and ${ }^{(e)}$ black hole angular momentum. All errors are provided at the $90 \%$ confidence level.

neutral iron. To test for the presence of an iron line, we focus on one spectral model: a power-law continuum observed behind ionised material partially covering the primary X-ray emitting region. In Fig. 4, we compare the data to the model (upper panel). We added to the spectral model a narrow Gaussian line with the energy fixed at $6.4 \mathrm{keV}$ in the rest frame of the quasar of fixed width $10 \mathrm{eV}$. The $\chi^{2}$ decreased by $\Delta \chi^{2}=10.8$ with one additional free parameter. Being aware of the problem of applying statistical test in this specific context (Protassov et al. 2002), an F-test formally infers a probability of $1.1 \%$ that the improvement reflects random chance. We conclude that neutral material reflecting X-rays in the direction of our line of sight is present.

To consistently describe the neutral iron fluorescence emission line and the associated reflection (contributing to the broad band, and specifically to the $2-5 \mathrm{keV}$ bump), we added a reflection component to the the fit. We modelled the reflection following Nandra et al. (2007). In this approach, the narrow line and the Compton reflection continuum are handled in a selfconsistent way. The model is based on the neutral reflection model of Magdziarz \& Zdziarski (1995), which assumes a slab geometry, and on the emission-line equivalent-width simulations of George \& Fabian (1991). In addition, the model considers the Compton shoulder according to the description of Matt (2002), and the $\mathrm{Fe} \mathrm{K} \beta$ and $\mathrm{Ni} \mathrm{K} \alpha$ lines with a flux of 11.3 per cent and 5 per cent of the iron $\mathrm{K} \alpha$, respectively. The model can be used within XSPEC under the name pexmon. Since we are unable to differentiate the reflection component from the continuum emission, we must minimise the number of free parameters, leaving only the reflection normalisation free to vary. Therefore, for all spectral modelling presented in this paper we fixed the pexmon photon index to the photon index of the power-law continuum. In addition we fixed the cut-off energy to $150 \mathrm{keV}$, the iron abundance to solar abundance, and the inclination angle to 45 degrees. The model was run such that only the reflected component was given, i.e., we set the relative reflectivity to be -1 .
We repeated the modelling of the spectra with the complex absorption scenarios assuming additional reflection by neutral material located far away from the X-ray emitting source. All models provide acceptable descriptions of the data and exhibit a $\chi^{2}$-value lower than found without reflection, where $\Delta \chi^{2}$ ranges from $\Delta \chi^{2}=18.5$ to $\Delta \chi^{2}=7.2$. About $10 \%$ to $20 \%$ of the "bump" emission can be attributed to the reflection component. The results for a partially covering warm absorber model are listed in Table 3 (fit 1), and in the lower panel of Fig. 4 we compare the data to the model.

\subsubsection{Reflection from an ionised accretion disk}

Following our previous results (Schartel 2007), we tested the possibility of describing the spectra assuming X-ray ionised reflection by the accretion disk. Our model consists of three additive components: (1) a power-law continuum, which describes the primary X-ray emitting source; (2) reflection by ionised material (Ross \& Fabian 2005; Crummy et al. 2005) blurred according to Dǒvciak, Karas \& Yaqoob (2004), which describes the disk emission; and (3) reflection by neutral material following Nandra et al. (2007), which allows us to account for possible reflection by material far away from the supermassive black hole. We assume that all three components are located behind a ionised, partially covering absorber.

To reduce the number of free parameters of the ionised reflection model, we tied the iron abundance of the ionised reflector to the iron abundance of the neutral reflection and the power-law index of the reflection model to the index of the primary continuum. Two parameters of the reflection model were allowed to vary: the ionisation level of the reflecting material and the normalisation. The convolution model of Dǒvciak et al. (2004) allows us to treat the black hole spin as a free parameter. Haardt's limb brightening is taken into account and the calculations are performed for 1000 grid points in the local energy frame, without renormalising the reflection component. 

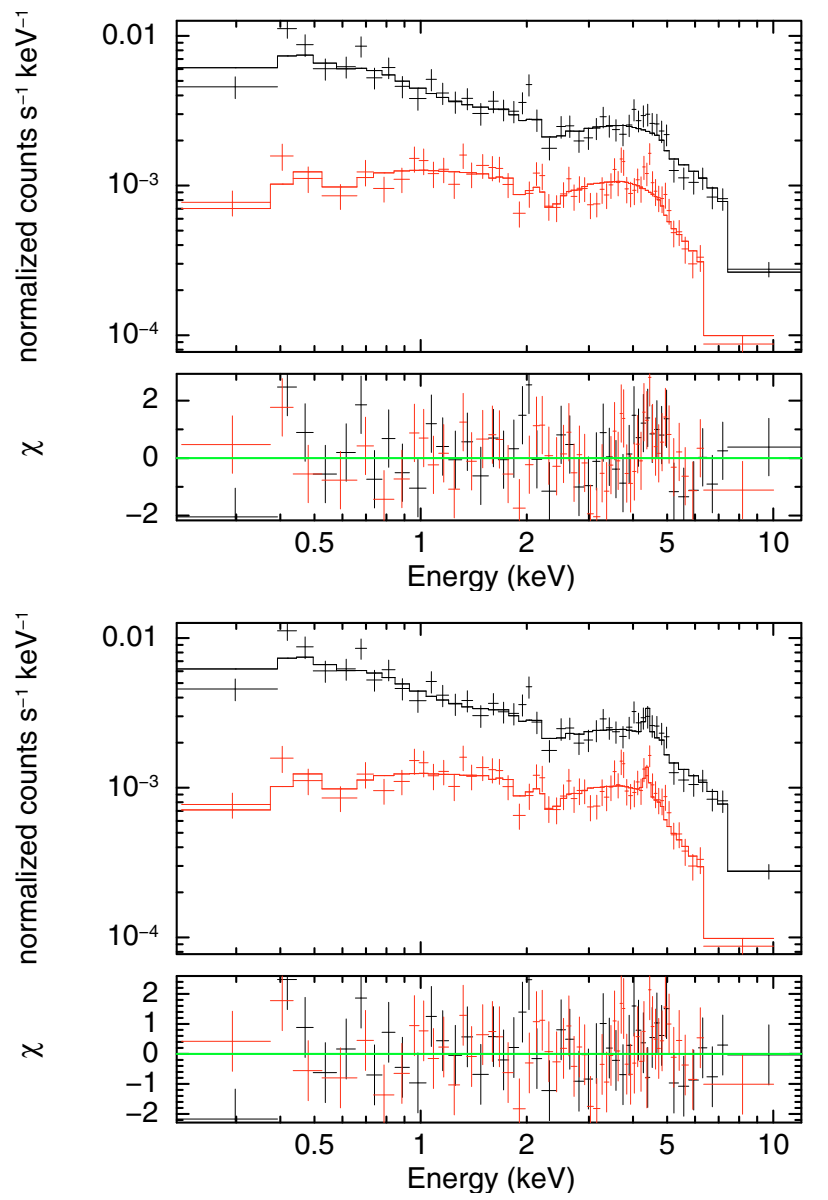

Fig. 4. The figure shows the two EPIC spectra of PG 2112+059 taken in May 2007. The black data points were taken with the pn camera, the grey (red in colour edition) with the MOSs. The spectra are modelled with a power-law continuum modified by a partially covering ionised absorber. Bottom panel shows the spectra in comparison to fit 1 listed in Table 3. It also includes a cold reflection component (continuum plus Fe K $\alpha$ line) parameterised by the pexmon model (Nandra 2007).

As we were unable to constrain the inner and outer radii of the emission region of the accretion disk, we fixed the inner radius to the lowest marginally stable orbit and the outer radius to $r_{\text {out }}=400 G M c^{-2}$. A trial without a neutral reflection component leaves residuals at the position of the redshifted iron line, as we found for the absorption models in the previous section, and therefore supports our model selection

The model allows a satisfying description of the data (e.g., fit 2 in Table 4). In Table 3, we list the best-fit values of the disk and black hole parameters derived by applying this model. Figure 5 compares the data to the best-fit model. The data are consistent with the assumption of a maximally rotating black hole.

\subsection{Spectra taken in November 2005}

Schartel et al. (2007) fitted only models of the disk assuming blurring according to Laor (1991). Therefore, to compare the two emission states, we modelled the EPIC spectra of PG 2112+059 taken in November 2005 assuming an ionised disk where the model is blurred according to Dǒvciak et al. (2004). Adding neutral reflection according to Nandra et al. (2007) does not improve the description of the data probably because of the poor statistics of the data. The statistics are also not good enough

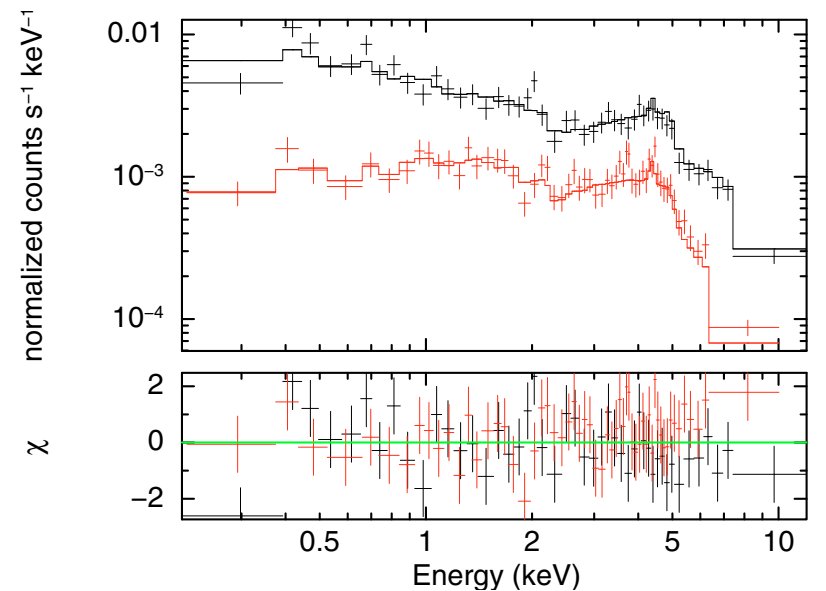

Fig. 5. The figure shows the pn (black) and MOS (grey,- red in colour edition) spectra of PG 2112+059 from May 2007 in comparison with a model (upper panel) and the corresponding residua (lower panel). The model assumes a power-law continuum, reflection on ionised material (Ross \& Fabian 2005) located in an accretion disk and reflection on neutron material far away from the primary X-ray emitting source (Nandra et al. 2007). The blurring was done following Dǒvciak et al. (2004). All three components are assumed to be located behind a ionised absorber. The best estimated parameters are provided in Tables 3 and 4 under fit 2 .

to constrain the inclination angle, the data being consistent with the assumption of a maximally rotating black hole.

\subsection{Spectra taken in November 2007}

The spectra taken in November 2007 yield a poor quality statistics and cannot be used to constrain the physical model. However, they are consistent with the ionised disk interpretation assuming blurring according to both Laor (1991) or Dǒvciak et al. (2004). The data are consistent with the assumption of a maximally rotating black hole.

\subsection{Joint fits to the spectra taken at different epochs}

From a mathematical viewpoint, the complex absorption models as well as the models that assume reflection by an ionised disk allow a satisfying description of the data when the states are analysed separately. We therefore studied whether the different scenarios allow a consistent description of the spectra observed at five different epochs. Since the source was observed in different states, our hope is that additional physical arguments can be found.

For each epoch, we fitted the pn spectrum and the combined MOS spectrum (obtained by adding the MOS1 and MOS2 spectra) in one fit, forcing all free and frozen parameters to have the same value for both data sets. Reflecting differences in the absolute calibration (Guainazzi 2009), the model for the MOS spectrum was multiplied with 1.07. All the models below include reflection from distant material, which was found to clearly be present.

\subsubsection{Absorption models}

A model consisting of both a power law and reflection by neutral material, where both components are partially covered by ionised material does not allow a satisfying description of the data $\left(\chi^{2}=599.5\right.$ for d.o.f. $\left.=246\right)$, although the covering fraction and the ionisation parameter were allowed to vary. Adding a 
Table 5. Joint fits to the EPIC spectra of PG $2112+059$ taken at different epochs: continuum properties.

\begin{tabular}{|c|c|c|c|c|c|c|c|c|c|c|}
\hline \multirow[t]{3}{*}{ Fit } & \multirow[t]{3}{*}{$\operatorname{Model}^{a}$} & \multicolumn{5}{|c|}{ Absorber } & \multirow{2}{*}{\multicolumn{2}{|c|}{$\begin{array}{l}\text { Continuum } \\
\text { power-law }\end{array}$}} & \multirow{3}{*}{$\begin{array}{c}\text { Distant } \\
\text { reflection } \\
\text { norm } \\
{\left[\mathrm{keV}^{-1} \mathrm{~cm}^{-2} \mathrm{~s}^{-1}\right]}\end{array}$} & \multirow{3}{*}{$\begin{array}{l}\text { Statistic } \\
\chi^{2} \quad d^{h}\end{array}$} \\
\hline & & & warm & & warm & & & & & \\
\hline & & \multicolumn{2}{|c|}{$\begin{array}{c}N_{\mathrm{H}}^{b} \\
{\left[10^{22} \mathrm{~cm}^{-2}\right]}\end{array}$} & $\begin{array}{r}\xi^{d} \\
{[\log ]}\end{array}$ & $\begin{array}{c}N_{\mathrm{H}}^{b} \quad \mathrm{cf}^{c} \\
{\left[10^{23} \mathrm{~cm}^{-2}\right]}\end{array}$ & $\begin{array}{r}\xi^{d} \\
{[\log ]}\end{array}$ & $\Gamma^{e}$ & $\begin{array}{c}\text { Norm }^{g} \\
{\left[\mathrm{keV}^{-1} \mathrm{~cm}^{-2} \mathrm{~s}^{-1}\right]}\end{array}$ & & \\
\hline \multirow[t]{6}{*}{3} & $i * i *(p+d)$ & \multirow{6}{*}{\multicolumn{2}{|c|}{$4.98_{-1.05}^{+0.95} 0.87_{-0.04}^{+0.03}$}} & & $3.47_{-1.09}^{+0.86} 0.71_{-0.09}^{+0.08}$ & & $2.22_{-0}^{+0}$ & & $2.43_{-0.86}^{+1.22} \times 10^{-4}$ & \\
\hline & & & & $+0.27_{-0.55}^{+0.40}$ & & $+3.07_{-0.24}^{+0.35}$ & & $9.40_{-1.80}^{+2.46} \times 10^{-5}$ & & \\
\hline & & & & $+1.50_{-0.16}^{+0.30}$ & & $+3.74_{-1.21}^{+2.26}$ & & $1.98_{-0.44}^{+0.53} \times 10^{-4}$ & & \\
\hline & & & & $-1.93_{-1.07}^{+0.99}$ & & $\begin{array}{r}+3.21_{-0.28}^{+0.55} \\
\text { + }\end{array}$ & & $3.89_{-0.92}^{+1.44} \times 10^{-5}$ & & \\
\hline & & & & $-0.68_{-0.35}^{+0.77}$ & & $+1.91_{-0.32}^{+0.09}$ & & $1.24_{-0.32}^{+0.49} \times 10^{-4}$ & & \\
\hline & & & & $-0.55_{-0.54}^{+0.97}$ & & $+1.83_{-0.63}^{+0.22}$ & & $8.70_{-2.27}^{+4.33} \times 10^{-5}$ & & 256.8243 \\
\hline & $*(p+k(r)+d)$ & $0.25_{-0.08}^{+0.09}$ & $1.00^{f}$ & & & & $1.65_{-0}^{+0}$ & & $1.91_{-0.91}^{+1.03} \times 10^{-5}$ & \\
\hline & & & & $-0.60_{-0.50}^{+0.47}$ & & & & $6.37_{-6.37}^{+3.30} \times 10^{-6}$ & & \\
\hline & & & & $\begin{array}{r}+1.86_{-0.80}^{+0.95} \\
\end{array}$ & & & & $5.97_{-0.67}^{+0.06} \times 10^{-5}$ & & \\
\hline & & & & $-0.79_{-0.31}^{+0.38}$ & & & & $0.00_{-0.00}^{+1.43} \times 10^{-6}$ & & \\
\hline & & & & $-0.98_{-0.26}^{+0.36}$ & & & & $3.27_{-1.90}^{+0.81} \times 10^{-6}$ & & \\
\hline & & & & $-1.09_{-0.29}^{+0.37}$ & & & & $2.13_{-2.13}^{+1.07} \times 10^{-6}$ & & 265.3234 \\
\hline
\end{tabular}

Notes. The first line of each modelling provides the best-fit model parameters of the joint fit. The second to the sixth line provide only the parameters that were free to vary between the spectra taken different epochs: September 2002 (Chandra observation of low state, second line), May 2003 (high state, third line), November 2005 (deep minimum state, fourth line), May 2007 (low state, fifth line), and November 2007 (low state, sixth line). Explanation of labels: ${ }^{(a)}$ the spectral models are described in Table 2; ${ }^{(b)}$ equivalent column density; ${ }^{(c)}$ covering fraction; (d) ionisation level of absorbing material $\xi=L / n r^{2}$, as defined in XSPEC; ${ }^{(e)}$ photon index of power-law continuum; ${ }^{(f)}$ fixed; ${ }^{(g)}$ normalisation of photon flux at $1 \mathrm{keV} ;{ }^{(h)}$ degrees of freedom. The best fitted parameters for the ionised reflector, the disk and the black hole are provided in Table 6. All errors are provided for the $90 \%$ confidence level.

Table 6. Joint fits to the EPIC spectra of PG 2112+059 taken at different epochs: ionised reflector, disk, and black hole properties.

\begin{tabular}{|c|c|c|c|c|c|c|c|}
\hline \multirow[t]{3}{*}{ Fit } & \multirow[t]{3}{*}{$M^{a}$} & \multicolumn{3}{|c|}{ Ionised Reflector } & \multicolumn{2}{|c|}{ Disk } & \multirow{3}{*}{$\begin{array}{c}\text { Black Hole } \\
\qquad a / M^{e}\end{array}$} \\
\hline & & Norm & $\mathrm{Fe}^{b}$ & $\Xi^{c}$ & Index $^{d}$ & Inclination & \\
\hline & & {$\left[\mathrm{cm}^{-2} \mathrm{~s}^{-1}\right]$} & {$\left[\mathrm{erg} \mathrm{cm} \mathrm{s}^{-1}\right.$ ] } & & [degree] & & \\
\hline \multirow[t]{6}{*}{4} & $k(r)$ & & $1.80_{-0.24}^{+0.26}$ & & & $52.5_{-2.3}^{+2.2}$ & $0.99_{-0.02}^{+0.0082}$ \\
\hline & & $8.35_{-5.08}^{+10.9} 10^{-6}$ & & $31.2_{-1.2}^{+8.2}$ & $4.45_{-0.63}^{+0.92}$ & & \\
\hline & & $8.77_{-4.10}^{+2.70} 10^{-7}$ & & $30.0_{-0.0}^{+13.2}$ & $3.00^{f}$ & & \\
\hline & & $2.96_{-1.46}^{+2.91} 10^{-7}$ & & $39.3_{-7.3}^{+9.7}$ & $2.87_{-0.21}^{+0.54}$ & & \\
\hline & & $6.55_{-2.06}^{+4.16} 10^{-7}$ & & $30.0_{-0.0}^{+3.5}$ & $2.89_{-0.20}^{+20}$ & & \\
\hline & & $4.13_{-2.45}^{+4.60} 10^{-7}$ & & $30.0_{-0.0}^{+7.0}$ & $2.79_{-0.39}^{+0.51}$ & & \\
\hline
\end{tabular}

Notes. The corresponding continuum properties are provided in Table 5. Explanation of labels: ${ }^{(a)}$ the spectral models are described in Table 2; ${ }^{(b)}$ iron abundance relative to solar iron abundance; ${ }^{(c)}$ ionisation parameter as defined in reflion; ${ }^{(d)}$ power-law dependence of emissivity; ${ }^{\left({ }^{)}\right)}$black hole angular momentum; ${ }^{(f)}$ fixed. All errors are provided for the $90 \%$ confidence level.

second layer of partially covering ionised material, we achieved a satisfying description of the data. The results are provided in Table 5 as fit 3 . We note that the description of the data by this model does not require any change in the covering fraction of the absorbing material.

In addition, we modelled the data allowing the covering fraction of both ionised absorbers to vary for the different source states. We obtain a $\chi^{2}=234.5$ for d.o.f. $=235$. An F-test shows that the model probably provides an improved description of the data, but below the 3- $\sigma$ threshold. The two absorption systems are characterised by $N_{\mathrm{H}} \sim 5 \times 10^{22} \mathrm{~cm}^{-2}$ and $N_{\mathrm{H}} \sim 2.3 \times 10^{23} \mathrm{~cm}^{-2}$. The ionisation parameters of the two systems do not show any separation. Especially, it is impossible to differentiate between a high and low ionisation system. In addition, the ionisation parameters do not indicate any correlation with the normalisation of the power-law continuum. Therefore, we do not discuss this model any further in the following.

\subsubsection{Reflection from an ionised accretion disk}

As in the previous section, we now describe how we jointly modelled the five spectra taken at different epochs assuming ionised reflection by the accretion disk that is blurred according to Dǒvciak et al. (2004), (see Sect. 5.1.3), seen behind a layer of ionised material. We verified that the modelling improves significantly by adding a reflection component from neutral material that is far away from the X-ray emitting source. The results of the analysis are provided in Tables 5 and 6 as fit 4 . 


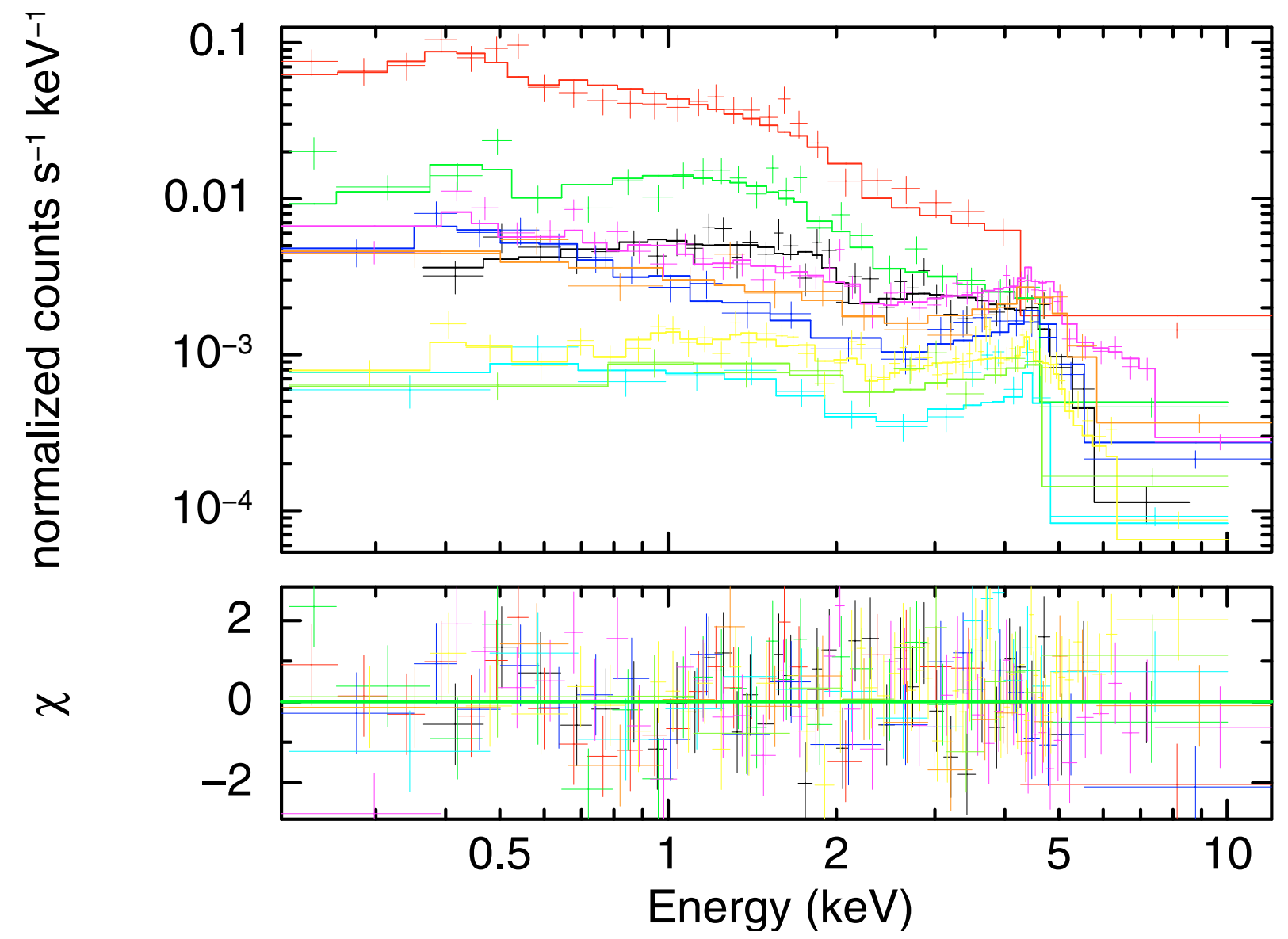

Fig. 6. The spectra of PG $2112+059$ at five different epochs (September 2002, intermediate state; May 2003, high state; November 2005, deep minimum state; May 2007, low state; and November 2007, low state) in comparison to a model consisting of a continuum power-law and a relativistically blurred X-ray ionised reflection component that originates in an accretion disk close to a supermassive black hole. Both components are observed through a warm absorber and reflected by neutral distant material (Tables 5 and 6, fit 4).

For the different epochs, the ionisation level of the warm absorber, the power-law continuum normalisation, the radial dependence of the emission of the disk, the ionisation level of the ionised reflector, and the normalisation of the emission of the ionised reflection were allowed to vary. We assumed that all spectra are observed through a warm absorber with the same column density. We also assume an identical power-law index, normalisation of the distant reflection component, inclination angle of the disk, and spin of the black hole. In addition, we fixed the radial dependence of the emission of the disk for the modelling of the highest flux state (May 2003) where it is basically unconstrained because of the low contribution of the reflection component to the total spectra. From a statistical viewpoint, the model allows us to describe the data with $\chi^{2}=266.6$ for d.o.f. $=234$. The observed spectra are compared with the model and the corresponding residuals in Fig. 6. No systematic residuals remain around the iron line complex.

\section{Discussion}

Along the lines of our original observational goal, we have successfully pinpointed PG $2112+059$ in its low state by acquiring a $\sim 25$ ks short snapshot observation, and we performed a long follow-up observation consisting of two $\sim 100 \mathrm{ks}$ XMM-Newton observations within two and a half weeks after the snapshot. Unfortunately, the two long observations were partly affected by enhanced background radiation. Nevertheless, the number of collected events is significantly higher than in previous data, allowing a much more detailed analysis of the source's low-state spectral features.

As illustrated in Fig. 3, the main scientific challenge is the interpretation of the excess emission or bump in the 2 to $5 \mathrm{keV}$ energy range. The request for consistency in the interpretation of the soft and hard parts of the spectra constrains the issue.

\subsection{Distant cold reflector}

The spectra of PG $2112+059$ taken in May 2007 exhibit a significant fluorescence line of neutral iron with a flux of $F=(6.4 \pm 2.8) \times 10^{-7}$ photons $\mathrm{cm}^{-2} \mathrm{~s}^{-2}$, which is in remarkable agreement with the flux found for a $(2.6-\sigma)$ line, $F=(5.0 \pm 3.2) \times 10^{-7}$ photons $\mathrm{cm}^{-2} \mathrm{~s}^{-2}$, detected in the November 2005 spectra (Schartel et al. 2007). The equivalent width found for the neutral iron line, $E W=110_{-40}^{+60} \mathrm{eV}$, is quite common for high luminosity, basically unabsorbed, type 1 objects. Jiménez-Bailón et al. (2005) detected fluorescence lines from neutral iron in $\sim 50 \%$ of the PG quasar sample, measure $\langle E W\rangle=80_{-20}^{+30} \mathrm{eV}$ with $\sigma_{E W}<40 \mathrm{eV}$. We interpret the line in the context of a distant reflector in the sense that the reflecting material is far away from the primary X-ray emitting source and the supermassive black hole. The distance is indeed so large that emission lines do not exhibit a measurable velocity broadening and the reflecting material does not become ionised. We accounted for the distance reflector following Nandra et al. (2007). 
The invariance of the line flux found in spectra of PG 2112+059 separated by 1.5 years supports this interpretation.

\subsection{Absorption}

The excess emission in the 2 to $5 \mathrm{keV}$ energy range can be described by a power-law source seen behind an absorber of high column density. Models with neutral absorbers estimate the column densities to be greater than $1.7 \times 10^{23} \mathrm{~cm}^{-2}$ for the spectra of PG 2112+059 taken in November 2005 (compare Table 3). Ionised material must be of significantly higher column densities for the bump to be modelled accurately. Guainazzi et al. (2005) found that the column density of obscured AGNs correlates well with the iron line equivalent width for $N_{\mathrm{H}}>10^{23} \mathrm{~cm}^{-2}$ and flattens at lower densities to an almost constant value of $E W=113 \pm 13 \mathrm{eV}$, which is in agreement with the values found for low luminosity unobscured quasars (Jiménez-Bailón et al. 2005). In the May 2007 spectra of PG $2112+059$, we detected a neutral iron emission line of $E W=110_{-40}^{+55} \mathrm{eV}$, which excludes models of the highest column densities considered.

All attempts to describe the spectra taken at different epochs, by assuming different flux levels for the primary X-ray emitting source, require two layers of absorbing materials of which at least one is ionised. The presented model (Table 5, fit 3) has two partially covering absorbing systems - a low ionised system with $N_{\mathrm{H}} \sim 5 \times 10^{22} \mathrm{~cm}^{-2}$ and $\xi=(0.01-30) \mathrm{erg} \mathrm{s}^{-1} \mathrm{~cm}$ and a highly ionised system with $N_{\mathrm{H}} \sim 3.5 \times 10^{23} \mathrm{~cm}^{-2}$ and $\xi=(70-5500) \mathrm{erg} \mathrm{s}^{-1} \mathrm{~cm}$. Both systems can be described with a constant covering fraction of $\mathrm{cf} \sim 0.8$.

The most similar absorbing system is that detected in NGC 1365 (Risaliti et al. 2005, 2009a,b). A system of four iron absorption lines demonstrates the presence of a highly ionised absorber, of column density $N_{\mathrm{H}} \sim 5 \times 10^{23} \mathrm{~cm}^{-2}$ (Risaliti et al. 2005). In addition, Risaliti et al. (2009b) observed a variable (low ionised or neutral) absorption system with $N_{\mathrm{H}} \sim 3.5 \times$ $10^{23} \mathrm{~cm}^{-2}$, which is probably a broad-line region cloud covering and uncovering the nucleus. Risaliti et al. (2009a) discussed two scenarios for explaining the observed variability. The one of special interest here consists of clouds with about the same size of the X-ray emitting source and assumes that, on average, few (1-3) of these clouds transverse the line of sight. Depending on the fluctuations in the number and column density of the clouds, the source exhibits a reflection-dominated or Comptonthin state.

The most striking difference between NGC 1365 and PG $2112+059$ is the amount of variability in the absorber, which is not detected at all for PG $2112+059$ as the data can be described by a constant covering fraction. However, the supermassive black hole mass of PG $2112+059$ is $\log M_{\mathrm{BH}} / M_{\text {solar }}=$ $9.0 \pm 0.1$ (Vestergaard \& Peterson 2006), which is a factor $>10$ more massive than the supermassive black hole of NGC 1365 $\left(\log M_{\mathrm{BH}} / M_{\text {solar }}=7.2-7.8 \pm 0.4\right.$, Risaliti et al. $\left.2009 \mathrm{~b}\right)$. As the geometry scales with black hole mass, an absorption system analogue to the system of NGC 1365 could scale its size by a factor of 10 . If we assume a similar size for the clouds, we may expect basically a constant number of clouds, $\sim 15$, along our line of sight, which would explain why we see no variability in the covering factor.

In Fig. 7, we show the ionisation parameter as a function of the power-law normalisation, which is proportional to the flux since the spectral slope is constant. The ionisation parameters of the low ionised absorber are plotted in black. The ionisation parameter is defined as $\xi=L n^{-1} r^{-2}$, where $n$ is the electron density, $L$ is the luminosity of the X-ray emitting source,

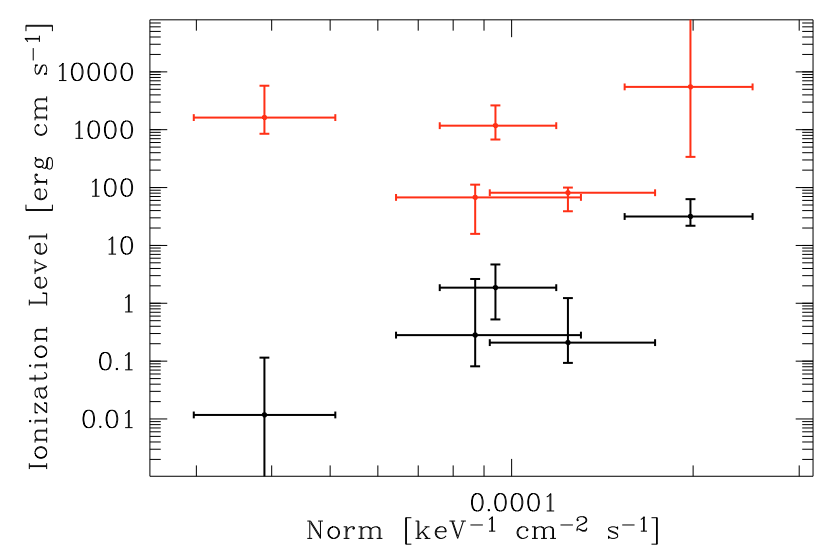

Fig. 7. The ionisation parameter is plotted against the power-law normalisation for the two partially covering absorption layers that allow a joint description of the spectra taken at different epochs and continuum flux levels (compare Table 5). As the power-law indices were tied to each other, the normalisation is directly equivalent to the flux. The highly ionised system is plotted in red and the low ionised system in black.

and $r$ is the distance. Given the considerations above, we can assume that the absorbing clouds are at a constant distance from the ionising source, which implies that $\xi \sim F n^{-1}$ where $F$ is the ionising flux. For a constant electron density, we expect that $\xi \propto F$. In Fig. 7 (black points), we find an increasing ionisation parameter with increasing flux. However, a flux increase by a factor of 5 is accompanied by an ionisation parameter increase of more than 250. An increased flux causes a higher ionisation level and consequently an increase in the electron density, which should even weaken the proportion between flux and ionisation parameter.

The highly ionised absorber of NGC 1365 (Risaliti et al. 2005) is probably located close to the supermassive black hole, hence the high value of its ionisation parameter. If this is the case, then the assumption of constant distance and density does not hold, complicating the relationship between $\xi$ and $F$. In Fig. 7, we do not detect a correlation between flux and ionisation parameter (red points). It could also be that the ions responsible for absorption are already fully ionised in the low flux state and therefore an increase in the flux would not affect the ionisation parameter.

Although the observed properties can be explained by a scenario similar and scaled according to the scenario developed by Risaliti et al. (2009a) for NGC 1365, there are very important differences from PG 2112+059. Risaliti et al. find tight constraints on the variability within individual observations and the absorption line identification is on a sound statistical basis. For PG 2112+059, the observations are separated by months and years, and there is no clear measurement of the flux state between the different observations. In addition, the statistical data are insufficient for tracing the variability in individual absorption lines.

\subsection{Ionised reflection from an accretion disk}

Ionised reflection from an accretion disk allows us to describe the high energy bump of the spectra taken of PG $2112+059$ in May 2007. Valid descriptions are obtained with the Laor (1991) blurring as well as the blurring according to Dorvciak et al. (2004). 
We can jointly describe all five spectra of PG $2112+059$ by assuming ionised reflection by an accretion disk blurred according to Dǒvciak et al. (2004), which is observed behind a screen of ionised material and emission from a distant neutral reflector. We find an ionisation parameter of the warm absorber that follows the flux of the continuum power laws (compare Fig. 8). Despite its large errors, the amplitude of the variation in the ionisation parameter is equal to the amplitude of the variation in the flux. With the exception of the high state, the index of the radial dependency of the disk emission agrees with the expected value of three. Hence, we consider this scenario to be physically meaningful and plausible.

\subsubsection{Spin of black hole}

The data taken in May 2007 allow us to estimate the angular momentum of the black hole, which is very high in all fits $(a / M>0.86)$ The data are consistent with a maximally rotating black hole. Jointly fitting the five spectra taken at different epochs does not allow us to place tighter constraints on the black hole spin.

\subsubsection{Iron abundance}

The models used to describe the warm absorber, the distant reflector, and the ionised reflection from the accretion disk include the iron abundance as a free parameter. We assumed that the iron abundance is the same in each of the spectral components. We note that the iron abundance of the absorption scenarios is within the range of abundances found for scenarios assuming ionised reflection by the disk when the blurring is modelled according to Dǒvciak et al. (2004), whereas the scenarios that treat blurring according to Laor (1991) show significantly higher abundance values. Restricting ourselves to the joint modelling of the spectra taken at different epochs, we find $\mathrm{Fe} / \mathrm{Fe}_{\odot}=1.5-2.2$ for the absorption scenarios, $\mathrm{Fe} / \mathrm{Fe}_{\odot}=1.6-1.9$ for the ionised reflection blurred according to Dǒvciak et al. (2004), and $\mathrm{Fe} / \mathrm{Fe}_{\odot}=$ 2.9-3.2 if blurred according to Laor (1991). These super-solar abundances are frequently measured in both low $-z$ and high- $z$ AGNs. However, the high metallicity value may be an artefact as different physical models in terms of limb brightening were applied.

\subsection{Accretion rate}

Witt et al. (1997) discussed an accretion disk with a hot continuous corona. The authors assumed that the corona itself accretes and is therefore powered by the release of the gravitational energy and cooled by radiative interaction with the disk. In this model, the radial infall is accompanied by a strong vertical outflow. In the context of our observations, the most important prediction is that at a given radius the corona forms only for accretion rates higher than a limiting value and that the fraction of the energy dissipated in the corona decreases with increasing accretion rate.

Considering optical and especially ultraviolet data we clearly trace a decrease in the flux from 2005 to 2007, which for the UVW2 filter is of the order of $10 \%$. The decrease of the $U$ band flux is of the order of $8 \%$, i.e., comparable with the variation found for the UV. Unfortunately, we do not have an ultraviolet observation that was performed during the high state in 2003. However, based on the estimated optical flux and assuming a constant ratio of optical to ultraviolet flux and accounting for

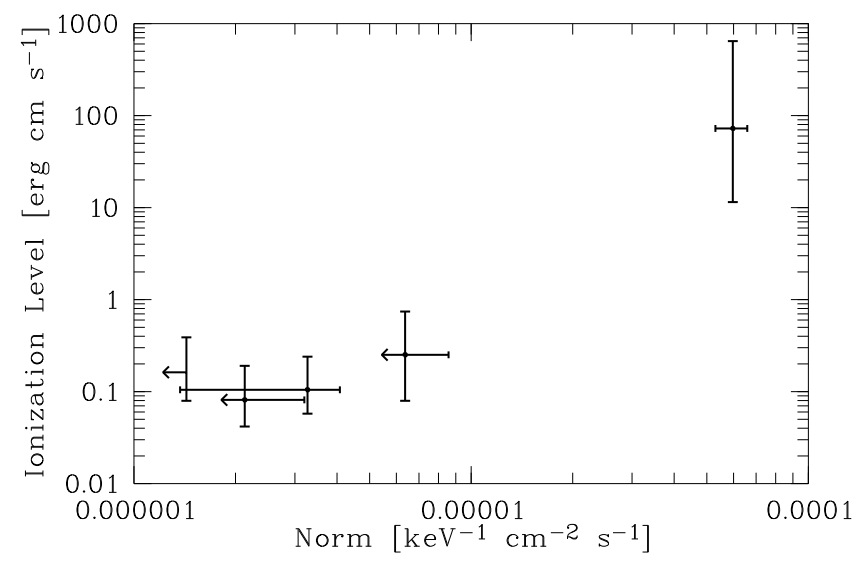

Fig. 8. The ionisation parameter of the warm absorber is shown against the power-law normalisation for the scenarios containing a Dóvciak, Karas \& Yaqoob accretion disk for an attempt to describe the spectra taken at different epochs and flux levels (compare Tables 5 and 6). As within each scenario, the power-law indices were tied to each other, the normalisation equals directly the flux.

our full error budget, we can assume for 2003 an UV flux not more that $20 \%$ higher than in 2005. Assuming that the ultraviolet emission is proportional to the accretion rate, we would expect a similar variability in the X-ray flux. However, the observed X-ray variability is larger than expected and, furthermore, in 2005, when the UV emission was $10 \%$ higher than in 2007, the X-ray count rate was about 40\% lower than in May 2007. Therefore, we cannot explain the X-ray variability with changes in the accretion rate using the model of Witt et al. (1997).

\subsection{Light bending model}

Our findings for PG $2112+059$ can be understood in the context of the light bending model for the spectral properties of accreting black holes developed by Miniutti \& Fabian (2004). In this model primary emission is generated by a ring-like source that emits hard X-ray radiation isotropically with constant luminosity in the form of a power law. The source is centred on a maximally rotating Kerr black hole rotation axis at a height of between only $1 r_{\mathrm{g}}$ and $20 r_{\mathrm{g}}$ above the equatorial plane. In this model, changes in the observed flux and changes in the ratio of the power law continuum to reflection from the disk are explained by variations in the height of the primary source: as the primary source lowers its height above the black hole, more light will be gravitationally bended towards the disk lowering the observed at infinity continuum flux, while the flux in the reflection increases.

The light bending model assumes a maximally spinning black hole. Modelling the spectra of PG $2112+059$ with the black hole spin as a free parameter we obtain very high estimates for the spin in agreement with the assumption of a maximally spinning black hole. The main arguments in favour of the Miniutti \& Fabian light bending model are the observed low states in 2005 and 2007. Blurring the disk reflection spectra according to Laor, the primary power-law components disappear completely, and we can even speak of "reflection-dominated" (Fig. 9). These spectra correspond to the regime 1 in the light bending model, where the primary source is located at only 2 to $4 r_{\mathrm{s}}$ from the black hole: the spectra are dominated by the reflection component and the continuum is strongly suppressed. The model of Laor blurring assumes a maximal rotating black hole. We obtain an index for the power law radial dependence of the disk emission significantly higher than 3 , which 

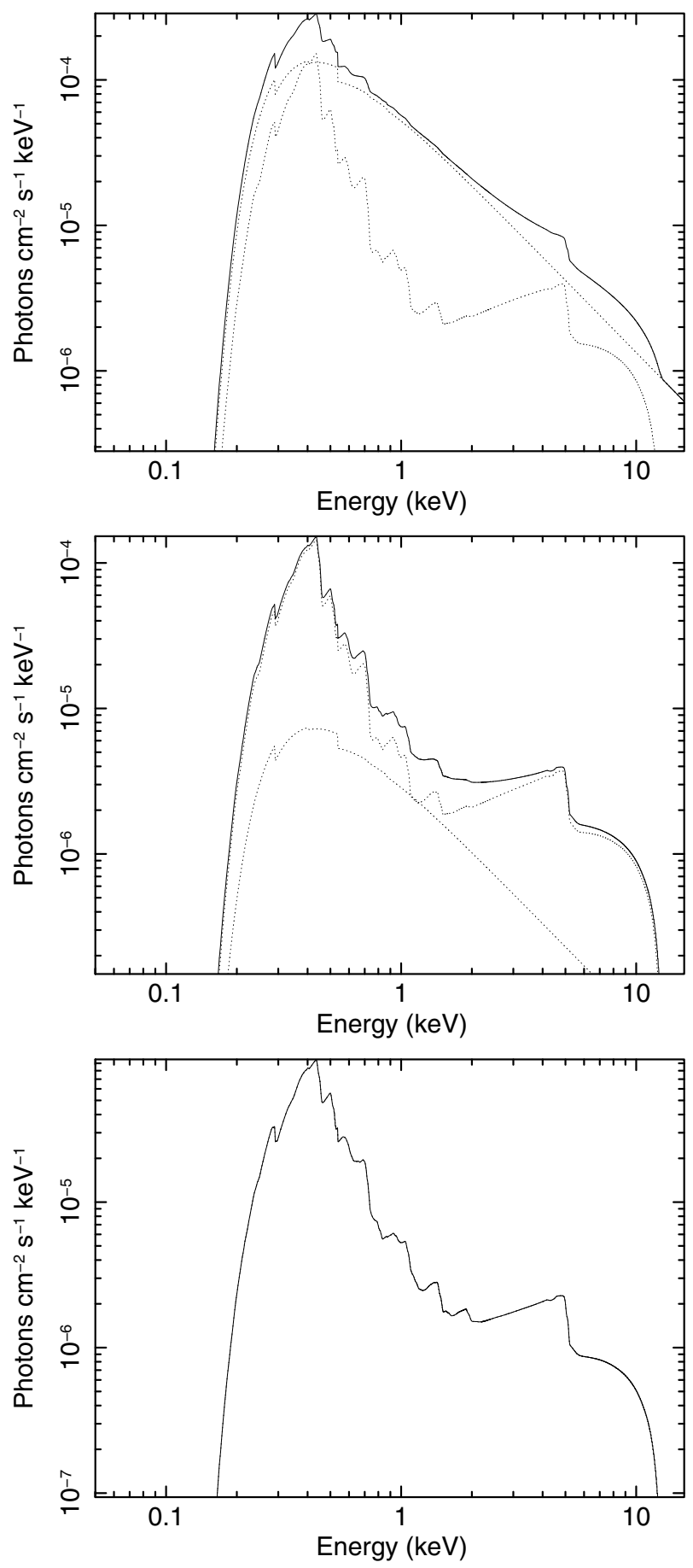

Fig. 9. The figure shows best-fit models assuming ionised reflection by the accretion disk at three epochs. The ionised absorber and the distant reflection components are not shown for clarity. The upper-panel shows the model for the May 2003 spectra, when the source showed the highest flux. The middle panel shows the model for the May 2007 spectra, when both components significantly contributed to the total emission. The lower panel shows the model for the May 2005, when PG 2112+059 was in a deep minimum state.

supports our interpretation as exactly this is predicted by the light bending model. The most accurately estimated disk inclination angle is in agreement with $60^{\circ}$, and therefore we can directly compare the measured flux ratios with Fig. 2 (lower panel) in Miniutti \& Fabian (2004). If we assume that the observed low states correspond to phase 1 of the model, then the observed continuum flux for the high state is in disagreement with the model assumptions.

Thus, light bending is the simplest, physically consistent model that can explain our observations of PG $2112+059$.

\section{Conclusions}

We have analysed observations that demonstrate that XMM-Newton can identify low states using a short snapshot observation and long follow-up observations. An absorption scenario, similar to the scenario proposed by Risaliti et al. (2009a) for NGC 1365 allows us to understand the X-ray spectra of PG $2112+059$ taken at different flux states. The system consists of two layers of ionised material, both covering the source by $80 \%$. They show column densities of $N_{\mathrm{H}} \sim 5 \times 10^{22} \mathrm{~cm}^{-2}$ and $N_{\mathrm{H}} \sim 3.5 \times 10^{23} \mathrm{~cm}^{-2}$. The first is moderately ionised and its ionisation levels follow the flux changes. The latter is highly ionised and shows not correlation with the flux of the source.

A scenario with reflection by an ionised disk in the context of a light bending model (Miniutti \& Fabian 2004) also provides a convincing physical interpretation of the different source states. The warm absorber ionisation is consistent with being correlated with the flux of the source, which provides an additional degree of self-consistency to the overall reflection-based model.

A joint analysis of spectra taken at different epochs (i.e., corresponding to different emission states) provide results that are useful to constraining the emission scenarios but do not allow us to break the degeneracy between complex absorption scenarios and reflection by an ionised disk in the case of PG $2112+059$. Future high resolution observations of PG $2112+059$ carried out by next-generation X-ray telescopes will be crucial to the detailed study of the absorption, the continuum shape, and the strength of the reflection components.

Acknowledgements. The work is based on observations obtained with XMM-Newton, an ESA science mission with instruments and contributions directly funded by ESA Member States and NASA. We thank K. Nandra for providing the pexmon model. Lucia Ballo acknowledges support from the Spanish Ministry of Science and Innovation through a "Juan de la Cierva" fellowship. Financial support for this work was provided by the Spanish Ministry of Science and Innovation, through research grant ESP2006-13608-C02-01. Enrico Piconcelli acknowledges support under ASI/INAF contract I/088/06/0. We thank the anonymous referee for many fruitful comments and suggestions.

\section{Appendix A: Exposure details}

In Table A.1, the exposure details for the XMM-Newton observations of PG $2112+059$ in 2007 are given. For each exposure identifier, we report the instrument, filter, start time, and exposure time.

\section{Appendix B: Processing details}

The EPIC and OM data were processesd as described in Schartel et al. (2007). The EPIC data were analysed to determine the time ranges with low radiation background level according to Piconcelli et al. (2005). Special care was taken to ensure that all data of each observation were screened and that the source and background counts were extracted in the same way such that artificial differences were avoided.

The source counts were extracted from a circular region of radius $r=20^{\prime \prime}$ centred on the source positions determined by eye for each observation and exposure. The eye-determined positions agree with the optical position of PG 2112+059, given the pointing accuracy of XMM-Newton of $\sim 1.5^{\prime \prime}$. 
Table A.1. XMM-Newton 2007 observations of PG 2112+059.

\begin{tabular}{|c|c|c|c|c|}
\hline$N .^{1}$ & $I^{2}$ & Filter & $\begin{array}{c}\text { Start } \\
\text { Day and time } \\
{[\mathrm{UT}]}\end{array}$ & Duration \\
\hline \multicolumn{5}{|c|}{ May 2007 / 5005006} \\
\hline 1 & M1 & thin 1 & 3 at $21: 45: 41$ & 24.1 \\
\hline 2 & M2 & thick & 3 at $21: 45: 41$ & 24.1 \\
\hline 3 & pn & thin 1 & 3 at $22: 08: 23$ & 22.1 \\
\hline 6 & $\mathrm{OM}$ & $V$ & 3 at $21: 50: 19$ & 2.2 \\
\hline 7 & $\mathrm{OM}$ & $U$-NoBar & 3 at $22: 32: 06$ & 2.2 \\
\hline 8 & $\mathrm{OM}$ & $B$ & 3 at $23: 13: 53$ & 2.2 \\
\hline 9 & $\mathrm{OM}$ & $V$ GRISM 2 & 4 at $23: 55: 40$ & 4.0 \\
\hline 10 & $\mathrm{OM}$ & $U V W 1$ & 4 at $01: 07: 27$ & 2.2 \\
\hline 11 & $\mathrm{OM}$ & UVM2 & 4 at $01: 49: 14$ & 2.2 \\
\hline 12 & $\mathrm{OM}$ & $U V W 2$ & 4 at $02: 31: 01$ & 2.8 \\
\hline 13 & $\mathrm{OM}$ & $U V$ GRISM 1 & 4 at $03: 22: 48$ & 3.8 \\
\hline \multicolumn{5}{|c|}{ May 2007 / 5005007} \\
\hline 1 & M1 & thin $\overline{1}$ & 19 at $10: 03: 47$ & 100.0 \\
\hline 2 & M2 & thick & 19 at $10: 03: 47$ & 100.0 \\
\hline 3 & pn & thin 1 & 19 at $10: 26: 29$ & 98.4 \\
\hline 6 & $\mathrm{OM}$ & V & 19 at $10: 08: 25$ & $2 \times 3.3$ \\
\hline 9 & $\mathrm{OM}$ & $U$-NoBar & 19 at $12: 08: 39$ & $2 \times 3.3$ \\
\hline 12 & $\mathrm{OM}$ & $B$ & 19 at $14: 08: 53$ & $2 \times 3.3$ \\
\hline 15 & $\mathrm{OM}$ & $V$ GRISM 2 & 19 at $16: 09: 07$ & $4 \times 5.0$ \\
\hline 19 & $\mathrm{OM}$ & $U V W 1$ & 19 at $22: 48: 45$ & $2 \times 3.3$ \\
\hline 20 & $\mathrm{OM}$ & $U V W 1$ & 20 at $00: 48: 59$ & 3.5 \\
\hline 21 & $\mathrm{OM}$ & UVM2 & 20 at $01: 52: 26$ & $3 \times 3.9$ \\
\hline 25 & $\mathrm{OM}$ & $U V W 2$ & 20 at $05: 23: 59$ & $3 \times 3.5$ \\
\hline 28 & OM & $U V$ GRISM 1 & 20 at $08: 34: 20$ & $2 \times 5.0$ \\
\hline 30 & OM & $U V$ GRISM 1 & 20 at $08: 34: 20$ & $2 \times 4.7$ \\
\hline \multicolumn{5}{|c|}{ May 2007 / 5005008} \\
\hline 1 & M1 & thin 1 & 21 at $11: 19: 23$ & 91.9 \\
\hline 2 & M2 & thick & 21 at $11: 40: 27$ & 90.6 \\
\hline 3 & pn & thin 1 & 21 at $11: 42: 58$ & 90.2 \\
\hline 6 & OM & $V$ & 21 at 10:00:35 & $2 \times 3.3$ \\
\hline 9 & OM & $U$-NoBar & 21 at $12: 00: 49$ & $2 \times 3.3$ \\
\hline 12 & $\mathrm{OM}$ & $B$ & 21 at $14: 01: 03$ & $2 \times 3.3$ \\
\hline 15 & $\mathrm{OM}$ & $V$ GRISM 2 & 21 at $16: 01: 17$ & $4 \times 5.0$ \\
\hline 19 & $\mathrm{OM}$ & UVW1 & 21 at $22: 40: 43$ & $2 \times 3.3$ \\
\hline 21 & $\mathrm{OM}$ & $U V W 1$ & 21 at $00: 40: 57$ & 3.5 \\
\hline 22 & $\mathrm{OM}$ & UVM2 & 22 at $01: 44: 24$ & $3 \times 3.7$ \\
\hline 25 & $\mathrm{OM}$ & $U V W 2$ & 22 at $05: 07: 39$ & $2 \times 3.5$ \\
\hline 29 & $\mathrm{OM}$ & $U V$ GRISM 1 & 22 at $08: 18: 00$ & 4.7 \\
\hline 30 & $\mathrm{OM}$ & $U V$ GRISM 1 & 22 at $08: 18: 00$ & 5.0 \\
\hline \multicolumn{5}{|c|}{ November 2007 / 5005009} \\
\hline 1 & M1 & thin 1 & 5 at $09: 52: 51$ & 91.9 \\
\hline 2 & M2 & thick & 5 at 09:52:51 & 90.6 \\
\hline 3 & pn & thin 1 & 5 at $10: 15: 12$ & 90.2 \\
\hline 6 & $\mathrm{OM}$ & $V$ & 5 at 09:57:29 & 2.0 \\
\hline 9 & $\mathrm{OM}$ & $U$-NoBar & 5 at $10: 35: 56$ & 2.5 \\
\hline 12 & $\mathrm{OM}$ & $B$ & 5 at $11: 22: 43$ & 2.0 \\
\hline 15 & $\mathrm{OM}$ & $V$ GRISM 2 & 5 at $12: 01: 10$ & $2 \times 4.0$ \\
\hline 19 & $\mathrm{OM}$ & $U V W 1$ & 5 at $14: 24: 44$ & $2 \times 3.5$ \\
\hline 23 & $\mathrm{OM}$ & $U V M 2$ & 5 at $16: 31: 38$ & $2 \times 3.5$ \\
\hline 26 & $\mathrm{OM}$ & $U V W 2$ & 5 at $18: 38: 32$ & $2 \times 3.5$ \\
\hline 29 & $\mathrm{OM}$ & $U V$ GRISM 1 & 5 at $20: 45: 26$ & 4.0 \\
\hline 30 & $\mathrm{OM}$ & $U V$ GRISM 1 & 5 at $21: 57: 13$ & 4.4 \\
\hline 31 & $\mathrm{OM}$ & $U V$ GRISM 1 & 5 at $23: 16: 17$ & 3.4 \\
\hline
\end{tabular}

Notes. ${ }^{(1)}$ Exposure identifier: If OM performed more than one exposure with the same specifications (filter, grism and exposure time) then only exposure identifier and start time of the first exposure is provided; (2) instrument, where M1 stands for MOS 1 and M2 for MOS2.

The screening for low background intervals was based on an annulus centred on the source position. For both cameras, we chose an inner radius of $r_{\text {inner }}=1^{\prime}$. Reflecting the different
Table B.1. Screening for low background level, source position and background extraction area.

\begin{tabular}{|c|c|c|c|}
\hline \multirow[t]{2}{*}{$N .^{1}$} & \multirow[t]{2}{*}{$\mathrm{EPIC}^{2}$} & \multicolumn{2}{|c|}{ Net Source } \\
\hline & & $\begin{array}{l}\text { Count rate } \\
{\left[10^{-2} \mathrm{~s}^{-1}\right]}\end{array}$ & $\begin{array}{r}\text { Exposure time } \\
{[\mathrm{ks}]}\end{array}$ \\
\hline \multicolumn{4}{|c|}{ Observation from $14.5 .2003 / \mathrm{OI}^{5}: 0150610201$} \\
\hline 1 & pn & $13.12 \pm 0.44$ & 7.1 \\
\hline 2 & M1 & $3.92 \pm 0.21$ & 8.9 \\
\hline 3 & M2 & $4.12 \pm 0.22$ & 9.2 \\
\hline \multicolumn{4}{|c|}{ Observation from 20.11.2005/ $\mathrm{OI}^{5}: 0300310201$} \\
\hline 1 & M1 & $0.39 \pm 0.03$ & 72.4 \\
\hline 2 & M2 & $0.37 \pm 0.03$ & 73.7 \\
\hline 3 & pn & $1.26 \pm 0.06$ & 63.7 \\
\hline \multicolumn{4}{|c|}{ Observation from 03.05.2007 / $\mathrm{OI}^{5}: 0500500601$} \\
\hline 1 & M1 & $0.65 \pm 0.06$ & 22.9 \\
\hline 2 & M2 & $0.54 \pm 0.06$ & 23.7 \\
\hline 3 & pn & $1.90 \pm 0.13$ & 13.8 \\
\hline \multicolumn{4}{|c|}{ Observation from 19.05.2007/ $\mathrm{OI}^{5}: 0500500701$} \\
\hline 1 & M1 & $0.55 \pm 0.04$ & 54.8 \\
\hline 2 & M2 & $0.54 \pm 0.03$ & 57.8 \\
\hline 3 & pn & $1.91 \pm 0.08$ & 40.4 \\
\hline \multicolumn{4}{|c|}{ Observation from 21.05.2007 / $\mathrm{OI}^{5}: 0500500801$} \\
\hline 1 & M1 & $0.68 \pm 0.03$ & 79.4 \\
\hline 2 & M2 & $0.54 \pm 0.03$ & 89.5 \\
\hline 3 & pn & $2.08 \pm 0.07$ & 62.8 \\
\hline \multicolumn{4}{|c|}{ Observation from 05.11.2007 / $\mathrm{OI}^{5}: 0500500901$} \\
\hline 1 & M1 & $0.43 \pm 0.03$ & 50.6 \\
\hline 2 & M2 & $0.45 \pm 0.03$ & 50.8 \\
\hline 3 & pn & $1.51 \pm 0.07$ & 42.5 \\
\hline
\end{tabular}

Notes. ${ }^{(1)}$ exposure identifier; (2) M1 stands MOS1 and M2 for MOS2; (3) background corrected source count rate in the energy range of $0.2-12.0 \mathrm{keV}$ for pn and $0.2-10.0 \mathrm{keV}$ for MOS, respectively; ${ }^{(4)} \mathrm{accu}-$ mulated exposure time; ${ }^{(5)}$ observation identifier.

detector geometry, we chose an outer radius of $r_{\text {outer }}=14^{\prime}$ for the MOS cameras and of $r_{\text {outer }}=11^{\prime}$ for the pn camera. The events were binned with $100 \mathrm{~s}$. We considered an energy range from 0.2 to $10 \mathrm{keV}$ for the two MOS cameras and from 0.2 to $12 \mathrm{keV}$ for the pn camera. Good time intervals were characterised by a count rate $C R<2.5 \mathrm{~s}^{-1}$ for MOS and $C R<5.0 \mathrm{~s}^{-1}$ for pn with the exception of the May 2003 pn observation, where we applied $C R<10.0 \mathrm{~s}^{-1}$ reflecting the high and soft background conditions.

The background spectra for the MOS cameras were collected from an annulus around the source position with an inner radius of $r_{\text {inner }}=40^{\prime \prime}$ and an outer radius of $r_{\text {outer }}=105^{\prime \prime}$. A circular region with a radius of $r=30^{\prime \prime}$ was used for the background determination for the pn spectra. The region was centred on about $\mathrm{RA}=21 \mathrm{~h} 14 \mathrm{~m} 49 \mathrm{~s}$ and Dec $=6 \mathrm{~d} 7 \mathrm{~m} 22 \mathrm{~s}$ for all observations with the exception of the November 2005 observation, where the centre was at RA $=21 \mathrm{~h} 14 \mathrm{~m} 57 \mathrm{~s}$ and Dec $=6 \mathrm{~d} 8 \mathrm{~m} 4 \mathrm{~s}$ reflecting the position angle of the observation.

In Table B.1 give the accumulated low background time for each exposure and the corresponding net source count rate.

\section{References}

Arnaud, K., Dorman, B., \& Gordon, D. 2009, XSPEC: An X-Ray Spectral Fitting Package, User's Guide for version 12.5.0, http://heasarc.gsfc. nasa.gov/xanadu/xspec/manual/manual.html

Anders, E., \& Ebihara, M. 1982, Geochim. Cosmoch. Acta, 46, 2363 
Avni, Y. 1976, ApJ, 210, 642

Ballo, L., Giustini, M., Schartel, N., et al. 2008, A\&A, 483, 137

Blustin, A. J., Dwelly, T., Page, M. J., et al. 2008, MNRAS, 390, 1229

Brandt, W. N., Laor, A., \& Wills, B. J. 2000, ApJ, 528, 637

Boroson, T. A., \& Green, R. F. 1992, ApJS, 80, 109

Brinkman, A. C., Behar, E., Güdel, M., et al. 2001, A\&A, 365, L324

Brinkmann, W., Papadakis, I. E., \& Ferrero, E. 2004, A\&A, 414, 107

Cardelli, J. A., Clayton, G. C., \& Mathis, J. S. 1989, ApJ, 345, 245

Crummy, J., Fabian, A. C., Brandt, W. N., et al. 2005, MNRAS, 361, 1197

Dǒvciak, M., Karas, V., \& Yaqoob, T. 2004, ApJS, 153, 205

Elvis, M. 2000, ApJ, 545, 63

Elvis, M., \& Fabbiano, G. 1984, ApJ, 280, 91

Fabian, A. C., Ballantyne, D. R., Merloni, A., et al. 2002, MNRAS, 331, L35

Fabian, A. C., Miniutti, G., Gallo, L., et al. 2004, MNRAS, 353, 1071

Fabian, A. C., Zoghbi, A., Ross, R. R., et al. 2009, Nature, 459, 540

Fan, L. L., Wang, H. Y., Wang, T., et al. 2009, ApJ, 690, 1006

Gallagher, S. C., Brandt, W. N., Laor, A., et al. 2001, ApJ, 546, 795

Gallagher, S. C., Brandt, W. N., Wills, B. J., et al. 2004, ApJ, 603, 425

Gallo, L. C. 2006, MNRAS, 368, 479

George, I. M., \& Fabian, A. C. 1991, MNRAS, 249, 352

Gibson, R. R., Brandt, W. N., \& Schneider, D. P. 2008, ApJ, 685, 773

Gibson, R. R., Brandt, W. N., Gallagher, S. C., et al. 2009, ApJ, 696, 924

Giustini, M., Cappi, M., \& Vignali, C. 2008, A\&A, 491, 425

Grupe, D., Schady, P., Leighly, K. M., et al. 2007, AJ, 133, 1988

Grupe, D., Komossa, S., \& Gallo, L. C. 2008a, ApJ, 681, 982

Grupe, D., Leighly, K. M., \& Komossa, S. 2008b, AJ, 136, 2343

Guainazzi, M., Matt, G., \& Perola, G. C. 2005, A\&A, 444, 119

Guainazzi, M. 2009, EPIC status of calibration and data analysis, Issue 2.7.4, XMM-SOC-CAL-TN-0018, http://xmm2.esac.esa.int/docs/ documents/CAL-TN-0018.pdf

Jansen, F., Lumb, D., Altieri, B., et al. 2001, A\&A, 365, L1

Jannuzi, B. T., Bahcall, J. N., Bergeron, J., et al. 1998, ApJSS, 118, 1

Jiménez-Bailón, E., Piconcelli, E., Guainazzi, M., et al. 2005, A\&A, 435, 449

Just, D. W., Brandt, W. N., Shemmer, O., et al. 2007, ApJ, 665, 1004

Kalberla, P. M. W., Burton, W. B., Hartmann, D., et al. 2005, A\&A, 440, 775

Kendall, M. G., \& Stuart, A. 1973, The advanced Theory of Statistics, Hafner,

New York, Sect. 19.26, 2, 97

Laor, A. 1991, ApJ, 376, 90

Laor, A., Fiore, F., Elvis, M., et al. 1997, ApJ, 477, 93

Leighly, K. M., Halpern, J. P., Jenkins, E. B., et al. 2007a, ApJ, 663, L103

Leighly, K. M., Halpern, J. P., Jenkins, E. B., \& Casebeer, D. 2007b, ApJS, 173, L1
Leighly, K. M., Hamann, F., Casebeer, D. A., \& Grupe, D. 2009, ApJ, 701, 176

Loiseau, N. 2007, User's Guide to the XMM-Newton Science Analysis System, Issue 4.1, http://xmm.esac.esa.int/external/xmm_user_support/ documentation/sas_usg/USG/USG.html

Magdziarz, P., \& Zdziarski, A. A. 1995, MNRAS, 273, 837

Mason, K. O., Breeveld, A., Much, R., et al. 2001, A\&A, 365, 36

Matt, G. 2002, MNRAS, 337, 147

Miniutti, G., \& Fabian, A. C. 2004, MNRAS, 349, 1435

Miniutti, G., Fabian, A. C., Brandt, W. N., et al. 2009, MNRAS, 396, L85

Nandra, K., O’Neill, P. M., George, I. M., et al. 2007, MNRAS, 382, 194

Piconcelli, E., Jiménez-Bailón, E., Guainazzi, M., et al. 2004, MNRAS, 351, 161

Piconcelli, E., Jiménez-Bailón, E., Guainazzi, M., et al. 2005, A\&A, 432, 15

Protassov, R., van Dyk, D. A., \& Connors, A. 2002, ApJ, 571, 545

Reeves, J., Done, C., Pounds, K., et al. 2008, MNRAS, 385, L108

Risaliti, G., Bianchi, S., Matt, G., et al. 2005, ApJ, 630, L129

Risaliti, G., Salvati, M., Elvis, M., et al. 2009a, MNRAS, 393, L1

Risaliti, G., Miniutti, G., Elvis, M., et al. 2009b, ApJ, 696, 160

Ross, R. R., \& Fabian, A. C. 2005, MNRAS, 358, 211

Schartel, N., Rodríguez-Pascual, P. M., Santos-Lleó, M., et al. 2005, A\&A, 433, 455

Schartel, N., Rodríguez-Pascual, P. M., Santos-Lleó, M., et al. 2007, A\&A, 474, 431

Schmidt, M., \& Green, R. F. 1983, ApJ, 269, 353

Spergel, D. N., Bean, R., \& Dor'e, O. 2007, ApJS, 170, 377

Steffen, A. T., Strateva, I., Brandt, W. N., et al. 2006, AJ, 131, 2826

Strateva, I. V., Brandt, W. N., \& Schneider, D. P. 2005, AJ, 130, 387

Strüder, L., Briel, U., Dennerl, K., et al. 2001, A\&A, 365, L18

Sulentic, J. W., Dultzin-Hacyan, D., Marziani, P., et al. 2006, Rev. Mex. Astron. Astrofis., 42, 23

Turner, M. J. L., Abbey, A., Arnaud, M., et al. 2001, A\&A, 365, L27

Véron-Cetty, M.-P., \& Véron, P. 200, A Catalogue of Quasars and Active Nuclei, 9th Edition, Scientific Report No. 19, European Southern Observatory, München

Verner, D. A., Ferland, G. J., Korista, K. T., et al. 1996, ApJ, 465, 487 Vestergaard, M., \& Peterson, B. M. 2006, ApJ 641, 689

Vignali, C., Brandt, W. N., \& Schneider, D. P. 2003, AJ, 125, 433

Vignali, C., Piconcelli, E., Bianchi, S., et al. 2008, MNRAS, 388, 761

Watson, M. G., Auguéres, J.-L., Ballet, J., et al. 2001, A\&A, 365, L51

Wang, T., Brinkmann, W., \& Bergeron, J. 1996, A\&A, 309, 81

Witt, H. J., Czerny, B., \& Życki, T. 1997, MNRAS, 286, 848

Zdziarski, A. A., Johnson, W. N., Done, C., et al. 1995, ApJ, 438, L63 